\title{
Doublecortin (DCX) Mediates Endocytosis of Neurofascin Independently of Microtubule Binding
}

\author{
Chan Choo Yap, ${ }^{1 \star}$ Max Vakulenko, ${ }^{1 \star}$ Kamil Kruczek, ${ }^{1}$ Bashir Motamedi, ${ }^{1}$ Laura Digilio, ${ }^{1}$ Judy S. Liu, ${ }^{2}$ \\ and Bettina Winckler ${ }^{1}$ \\ ${ }^{1}$ Department of Neuroscience, University of Virginia, Charlottesville, Virginia 22908, and ${ }^{2}$ Center for Neuroscience Research, Children's National Medical \\ Center, Washington DC 20010-2970
}

Doublecortin on X chromosome (DCX) is one of two major genetic loci underlying human lissencephaly, a neurodevelopmental disorder with defects in neuronal migration and axon outgrowth. DCX is a microtubule-binding protein, and much work has focused on its microtubule-associated functions. DCX has other reported binding partners, including the cell adhesion molecule neurofascin, but the functional significance of the DCX-neurofascin interaction is not understood. Neurofascin localizes strongly to the axon initial segment in mature neurons, where it plays a role in assembling and maintaining other axon initial segment components. During development, neurofascin likely plays additional roles in axon guidance and in GABAergic synaptogenesis. We show here that DCX can modulate the surface distribution of neurofascin in developing cultured rat neurons and thereby the relative extent of accumulation between the axon initial segment and soma and dendrites. Mechanistically, DCX acts via increasing endocytosis of neurofascin from soma and dendrites. Surprisingly, DCX increases neurofascin endocytosis apparently independently of its microtubule-binding activity. We additionally show that the patient allele DCXG253D still binds microtubules but is deficient in promoting neurofascin endocytosis. We propose that DCX acts as an endocytic adaptor for neurofascin to fine-tune its surface distribution during neuronal development.

\section{Introduction}

Many of the proteins crucial for neuronal functioning are spatially segregated to distinct functional domains (Horton and Ehlers, 2003; Salzer, 2003; Lasiecka et al., 2009). During development, cell adhesion molecules such as the L1-CAM family members (L1, neurofascin, NrCAM) regulate axon outgrowth and are enriched on the growing axon (Maness and Schachner, 2007). In addition, neurofascin (NF) and NrCAM are prominently enriched on and play roles at the axon initial segment (AIS) and Nodes of Ranvier at later times. NF is an essential gene, and knockout mice lack Nodes of Ranvier (Sherman et al., 2005). Even though NF is not required for assembly of the AIS, it is necessary for maintaining it (Zonta et al., 2011). NF plays additional roles during development, but these roles are less well understood. For instance, NF has been implicated in GABAergic synaptogenesis at the AIS (Ango et al., 2004; Burkarth et al., 2007;

Received Oct. 20, 2011; revised March 6, 2012; accepted April 1, 2012.

Author contributions: C.C.Y., M.V., and B.W. designed research; C.C.Y., M.V., K.K., and B.M. performed research; J.S.L. contributed unpublished reagents/analytic tools; C.C.Y., M.V., K.K., B.M., L.D., and B.W. analyzed data; B.W. wrote the paper.

We thank Drs. Heike Folsch and Kevin Pfister for critical reading of the manuscript. We are grateful to Drs. Alex Benmerah, Gregor Eichele, Matt Rasband, Vann Bennett, Vance Lemmon, Bryce Paschal, Ira Mellman, and Kevin Pfister for generously providing crucial reagents. Lastly, we thank all members of the Winckler Laboratory. We thank Joshua Katz and Sutirtha Datta for initial help with experiments. K.K. is from the Jagiellonian University, Program in Biotechnology, Cracow, Poland.

*C.C.Y. and M.V. contributed equally to this work.

The authors declare no competing financial interests.

Correspondence should be addressed to Bettina Winckler, University of Virginia, Department of Neuroscience, 409 Lane Road, Charlottesville, VA 22908. E-mail: BWinckler@virginia.edu.

DOI:10.1523/JNEUROSCI.5318-11.2012

Copyright $\odot 2012$ the authors $\quad 0270-6474 / 12 / 327439-15 \$ 15.00 / 0$
Kriebel et al., 2011) and can affect neurite outgrowth of cultured neurons (Volkmer et al., 1996; Pruss et al., 2004, 2006).

It is well established that ankyrinG (ankG) anchors receptors at the AIS (for review, see Ogawa and Rasband, 2008). For example, a point mutation in the FIGQY ankyrin-binding motif of NF led to loss of AIS localization (Boiko et al., 2007; Dzhashiashvili et al., 2007). Importantly, the FIGQY motif bound strongly to ankyrinG only when nonphosphorylated (Tuvia et al., 1997; Zhang et al., 1998). When phosphorylated downstream of neurotrophin signaling, the FIGQY motif bound to doublecortin (DCX) instead (Kizhatil et al., 2002). DCX is mutated in patients with lissencephaly ("smooth brain"), a neurodevelopmental disease with layering defects in the cortex and hippocampus that result in cognitive deficits and epilepsy (des Portes et al., 1998; Gleeson et al., 1998). In addition, DCX also functions in later stages of neurodevelopment (Friocourt et al., 2003; Schaar et al., 2004; Deuel et al., 2006; Kappeler et al., 2007), such as axon and dendrite formation (Deuel et al., 2006; Cohen et al., 2008). On the molecular level, DCX binds microtubules via binding interfaces contained within two doublecortin (DC) repeats. Interestingly, most of the known human mutations in DCX are contained within these repeats. The phenotypes associated with DCX mutations or depletion are therefore postulated to be due to impairment of microtubule-related functions (Sapir et al., 2000; Kim et al., 2003; Leger et al., 2008; Liu, 2011). In addition to microtubule binding, DCX also binds several other proteins (Caspi et al., 2000; Tanaka et al., 2004), such as clathrin adaptors (Friocourt et al., 2001) and NF (Kizhatil et al., 2002). It is not known whether the interaction of DCX with NF affects either NF localization or function. 
In this work, we therefore investigated whether DCX plays a role in NF trafficking. We found that DCX promotes NF endocytosis from soma and dendrites in developing neurons and thereby modulates the surface distribution of NF. Importantly, this novel role of DCX is independent of microtubule binding. This finding suggests the possibility that DCX has microtubuleindependent functions in neurodevelopment.

\section{Materials and Methods Reagents \\ Antibodies}

Anti-NF. Anti-Pan-neurofascin against an extracellular epitope, mouse clone A12/18, and anti-Pan-neurofascin against an intracellular epitope, mouse clone L11A/41, were obtained from University of California, Davis, CA (NIH NeuroMab facility).

Anti-VGNC. Anti-pan-VGNC (voltage-gated sodium channel) mouse monoclonal antibody was obtained from Sigma.

Anti-DCX. Rabbit polyclonal anti-DCX antibody was from Cell Signaling Technology. Rabbit polyclonal anti-DCX antibody was from Abcam.

Anti-HA. The mouse monoclonal antibody was from Covance, and the rabbit polyclonal antibody was from Santa Cruz Technology.

Anti-FLAG. Mouse and rabbit anti-FLAG antibodies were from Sigma.

Anti-GFP. Mouse and rabbit anti-GFP antibodies were from Invitrogen.

Anti-ankyrinG. Rabbit anti-ankyrinG was described previously (Boiko et al., 2007). Mouse anti-ankyrinG, cloneN106/36 from University of California, Davis, CA/NIH NeuroMab Facility.

Anti-tubulin. Mouse monoclonal anti-tubulin was from Sigma.

Phalloidin. Palloidin was from Invitrogen.

Rabbit anti-phospho-FIGQY. Anti-phospho-FIGQY was kindly provided by Dr. Vann Bennett, Duke University, Durham, NC (Kizhatil et al., 2002).

Anti-L1. Rabbit anti-L1 antibody was generously provided by Vance Lemmon, University of Miami, Miami, FL.

Anti-LAMP1. Rabbit serum Mingus was from Ira Mellman (Genentech).

Anti-EEA1. Mouse anti-EEA1 antibody was from BD Transduction Laboratories.

Anti-NgCAM. Clone 8D9 was from the NIH Developmental Studies Hybridoma Bank, University of Iowa, Iowa City, IA.

Secondary antibodies. Alexa Fluor dye-coupled antibodies were obtained from Invitrogen. Horseradish peroxidase-coupled secondary antibodies were obtained from GE Healthcare.

\section{shRNA plasmids}

To specifically knockdown the DCX protein expression, we used validated short hairpin (sh) RNA targeting the rat DCX sequences obtained from Super Array Bioscience. The shRNAs were expressed from the U1 promoter cassette of a pGeneclip hMGFP vector containing GFP under the regulation of the CMV promoter. The sequences of the four short hairpin RNA targeting the rat DCX were: shDCX\#1: AGG CCA AGA AGG TAC GTT TCT; shDCX\#2: TCA CCT GTC TCC ATG ATT TCT; shDCX\#3: AAG GTT GAG TAT ACC AAG AAT; shDCX\#4: GTC AGC TCT CAA CAC CTA AGT. The scrambled sequence used as a control (shRandom) was GGA ATC TCA TTC GAT GCA TAC.

\section{Plasmids}

DCX plasmid. Mouse wild-type DCX-GFP and FLAG-DCX were kind gifts of Dr. Gregor Eichele, Max Planck Institute for Biophysical Chemistry, Göttingen, Germany (Tsukada et al., 2003).

Eps15 plasmid. All Eps15-GFP constructs were a kind gift from Dr. Alex Benmerah (Benmerah et al., 1999).

HA-neurofascin. HA-neurofascin was described previously (Boiko et al., 2007).

Lamin-cherry. Lamin-cherry was a kind gift from Dr. Bryce Paschal, University of Virginia, Charlottesville, VA.

Rab11-GFP was a kind gift from Dr. Ira Mellman (Genentech).

\section{Cell culture}

\section{Hippocampal neurons}

Primary cultures of hippocampal neurons were obtained from embryonic day 18 (E18) rat hippocampi of either sex as described (Yap et al., 2008a). Cells were plated on poly-L-lysine coverslips and incubated with DMEM medium with $10 \%$ horse serum. After $4 \mathrm{~h}$, the cells were transferred into serum-free medium supplemented with B27 (Invitrogen) and cultured for 9-12 days in vitro (DIV). Our hippocampal cultures contained astrocytes and other non-neuronal cell types, but neurotrophins were not added exogenously.

PC12 cells

PC12 cells were maintained in DMEM with 10\% horse serum and 5\% fetal bovine serum, and all transfections were conducted using Lipofectamine 2000 (Invitrogen) according to the manufacturer's protocol. NGF (from Sigma) was used at $100 \mathrm{ng} / \mathrm{ml}$ for $18-24 \mathrm{~h}$.

\section{COS cells}

COS cells were maintained in DMEM plus 10\% fetal bovine serum, and all transfections were conducted using Lipofectamine 2000 (Invitrogen) according to the manufacturer's protocol.

\section{Transfection of neuronal cultures}

Neuronal cultures at DIV 9-12 were transfected using Lipofectamine 2000 with $1 \mu \mathrm{g}$ of DNA, $3 \mu \mathrm{l}$ of Lipofectamine 2000 for $60-90 \mathrm{~min}$, and washed and incubated for $20 \mathrm{~h}$ as described previously (Yap et al., 2008b). Nucleofection of freshly dissociated neurons was performed using a Nucleofector device (Amaxa Biosystems) according to the manufacturer's instructions.

\section{Immunocytochemistry}

Cells were fixed in 2\% paraformaldehyde/ $3 \%$ sucrose/PBS in $50 \%$ conditioned medium at room temperature for $30 \mathrm{~min}$ and quenched in 10 $\mathrm{mm}$ glycine/PBS for $10 \mathrm{~min}$. The fixation conditions used do not introduce holes into the overwhelming majority of cells. Coverslips were then blocked in $5 \%$ horse serum $/ 1 \%$ BSA/PBS $\pm 0.05 \%$ Triton X-100 for 30 $\mathrm{min}$. Antibodies were diluted in 1\% BSA/PBS and incubated for $1-2 \mathrm{~h}$, essentially as described previously by Wisco et al. (2003). Coverslips were mounted in Vectashield (Vector Laboratories) and viewed on a Zeiss Axiophot with a $40 \times$ objective. Images were captured with the Orca cooled CCD camera (Hamamatsu) using Openlab software (ImproVision) and processed identically in Adobe Photoshop.

\section{Generation of DCX-GFP mutants}

\section{Point mutations}

Point mutations were introduced using QuikChange site-directed mutagenesis from Stratagene. All mutations were confirmed by sequencing.

\section{FLAG-DCX-R2}

Full-length mouse cDNA of DCX resistant to shDCX\#2 was generated by

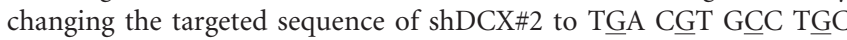
ACG AㄷT TTT by site-directed mutagenesis (Stratagene). The resistant full-length DNA was cloned into pCMV-Tag2 vector (Stratagene) at PstI and XhoI sites to generate PCMV-FLAG-DCX-R2 plasmid.

\section{Duolink assay}

The doublecortin/neurofascin proximity was detected in situ using the Duolink II fluorescence kit according to the manufacturer's instructions (Olink Bioscience). Briefly, cultured neurons were fixed and permeabilized and primary antibodies against doublecortin and neurofascin were applied under standard conditions. Duolink secondary antibodies conjugated to oligonucleotides were then added and incubated at $37^{\circ} \mathrm{C}$ for $1 \mathrm{~h}$ in a preheated humidity chamber. This was then followed by ligation and amplification using ligase and polymerase solution that also contained fluorescently labeled oligonucleotides. Signal is only generated if the two antigens are within about $40 \mathrm{~nm}$ of each other. After completing the Duolink assay, standard fluorescently labeled secondary antibodies were applied to stain doublecortin and neurofascin. 
A

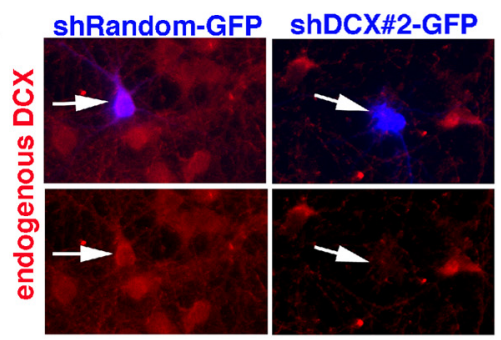

C

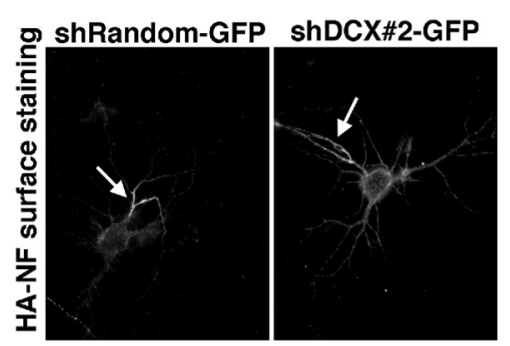

E

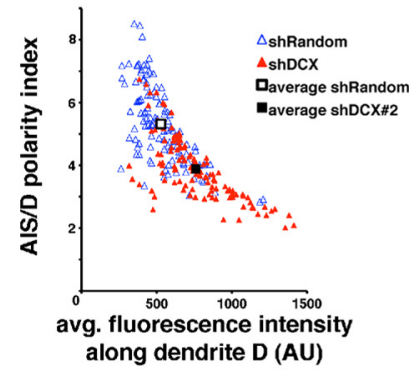

B

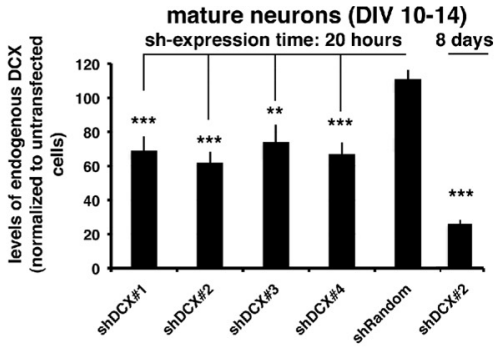

D

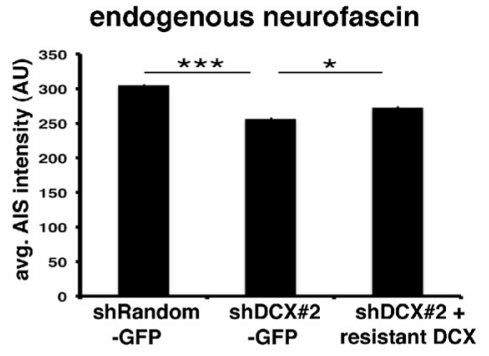

$\mathbf{F}$

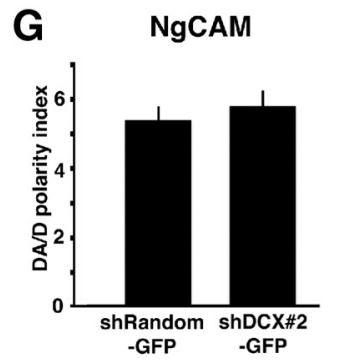

H

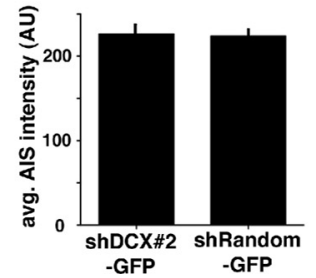

HA-NF localization

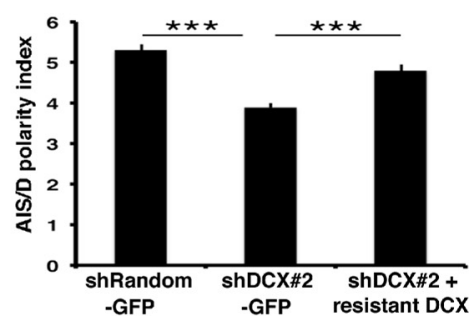

I endogenous ankyring

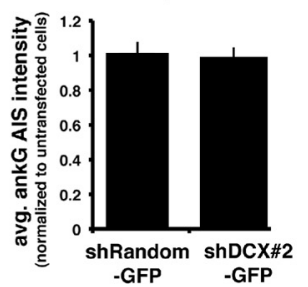

Figure 1. Downregulating DCX levels affects neurofascin surface distribution between dendrites and the AIS. $A$, Cultured neurons (DIV9/10) were transfected with shRandom-GFP (left panels) or shDCX\#2-GFP (right panels; blue) for $20 \mathrm{~h}$ and then stained against endogenous DCX (red). Single-channel images of the DCX staining are shown in the bottom panels. The transfected cell is indicated with an arrow. $\boldsymbol{B}$, Mature, cultured neurons were transfected with shRandom-GFP or four different shDCX-GFP plasmids and then stained against endogenous $D C X$. Levels of endogenous $D C X$ were determined from immunofluorescence images and normalized to untransfected cells. The extent of downregulation was determined after $20 \mathrm{~h}$ of shRNA expression in DIV10 cells to parallel experiments using acute HA-NF expression, or after 7-8d of shRNA expression in DIV14 cells to parallel experiments with endogenous proteins. ${ }^{* *} p<0.0001 ;{ }^{* *} p<$ 0.001 ; Bars, SEM. C, Surface HA-NF was stained in cells expressing HA-NF and either shRandom-GFP as control (left panel) or shDCX\#2-GFP (right panel) for $20 \mathrm{~h}$. Arrow indicates AIS.D, AIS intensity was determined for endogenous neurofascin (DIV14, plasmid expression for $7 \mathrm{~d}$ ) after transfection with shRandom, shDCX\#2, or shDCX\#2 with resistant DCX. $N=110$ cells shRandom, 94 cells shDCX\#2, 83 cells shDCX\#2 plus resistant $D C X .{ }^{* * *} p<0.0001 ;{ }^{*} p<0.01$; Bars, SEM. E, F, Quantification of HA-NF surface staining on AIS versus dendrites (AIS/D) in cells expressing shRandom-GFP, shDCX\#2-GFP, or shDCX\#2-GFP with an shRNA-resistant DCX (DIV9/10, plasmid expression for $20 \mathrm{~h}$ ). Scatter plot $(\boldsymbol{E})$ of dendrite intensity and HA-NF AIS/D polarity index for individual cells transfected with shRandom-GFP (blue) or shDCX (red) for $20 \mathrm{~h}$; AU, Arbitrary units. Average values from all cells are displayed as squares and are graphed in the histogram in $(\boldsymbol{F})$ for the AIS/D polarity index. $N=113$ cells shRandom, 113 cells shDCX\#2, 121 cells shDCX\#2 + resistant DCX. ${ }^{* * *} p<0.0001$; Bars, SEM. G-I, Effects of DCX downregulation in neurons on two other membrane proteins and the cytoskeletal AIS component ankG were quantified: exogenously expressed NgCAM (expression for $20 \mathrm{~h}$, fixed DIV10) (G), endogenous voltage-gated sodium channel (VGSC) (expression for $7 \mathrm{~d}$, fixed DIV14) $(\boldsymbol{H}$ ), and ankyring (expression for 7 d, fixed DIV14) (I). NgCAM: $n=33$ cells shRandom, $n=25$ cells shDCX; VGSC: $n=22$ cells shRandom, $n=24$ cells shDCX; AnkyrinG: $n=30$ cells shRandom, $n=23$ cells shDCX.

\section{Endocytosis assay}

Neurons

Neurons expressing HA-neurofascin for $20 \mathrm{~h}$ were incubated with rabbit anti-HA antibodies for $30 \mathrm{~min}$ at $37^{\circ} \mathrm{C}$ and washed several times, and any antibody remaining on the surface was stripped by treatment with MEM, pH 2.0, for 2 min (Fourgeaud et al., 2003), washed extensively, and returned to the incubator for various amounts of times before fixation in $2 \%$ paraformaldehyde/ $3 \%$ sucrose/PBS, pH 7.4. Surface HA-NF was visualized with mouse anti-HA antibody and Alexa Fluor 488 goat anti-mouse secondary antibody. Internalized anti-HA antibody was detected by applying Alexa Fluor 568 goat anti-mouse antibody after permeabilization. Permeabilization was achieved with $0.2 \%$ Triton X-100 for $10 \mathrm{~min}$ at room temperature as by Wisco et al. (2003). For neurons transfected by nucleofection, endocytosis assay was done at DIV3 by labeled live with mouse anti-NF.

\section{PC 12 cells}

For endocytosis experiments, PC-12 cells were plated in 12-well plates onto polylysine-coated coverslips until confluent in feeding medium ( $10 \%$ horse serum, $5 \%$ FBS, and $1 \%$ Na pyruvate in DMEM). Transfections were carried out with Lipofectamine 2000 (0.6 $\mu \mathrm{g}$ of DCX/ $0.4 \mu \mathrm{g}$ of neurofascin plasmids) for $45 \mathrm{~min}$. Lipofectamine was washed away and cells were changed into differentiating medium ( $1 \%$ horse serum, $1 \%$ fetal calf serum, and $1 \%$ sodium pyruvate in DMEM) containing NGF at $100 \mathrm{ng} / \mathrm{ml}$. Endocytosis assays were performed 24-48 h later. For endocytosis assays, medium was replaced with medium containing 1:200 rabbit anti-HA antibody for $20 \mathrm{~min}$ in $37^{\circ} \mathrm{C}$ with $250 \mathrm{ng} / \mathrm{ml} \mathrm{NGF}$. Acid strip and surface labeling was carried out as described for neurons (see Endocytosis assay).

\section{Immunoprecipitation procedure}

From HEK293 cells

For immunoprecipitations, transfected HEK293 cells were lysed for $45 \mathrm{~min}$ on ice in buffer containing 20 mM Tris-HCl ( pH 7.4), 2 mM EDTA, 2 mм EGTA, 0.1 mм DTT, 100 mм KCl, 10 mм NaF, 1 mm PMSF, 1\% Triton X-100 and Complete Protease Inhibitor Cocktail. Lysed cells were centrifuged at 12,000 rpm for $15 \mathrm{~min}$, and the supernatants were then precleared with protein G Sepharose (GE Healthcare) at $4^{\circ} \mathrm{C}$ for $3 \mathrm{~h}$. Precleared lysates were immunoprecipitated with mouse anti-HA or mouse antiGFP for $1 \mathrm{~h}$ followed by overnight incubation at $4{ }^{\circ} \mathrm{C}$ with protein $\mathrm{G}$ Sepharose. Immunoprecipitates were washed $5 \times$ with lysis buffer containing $0.1 \%$ Triton X-100 and eluted by 150 $\mathrm{mm}$ Tris- $\mathrm{HCl}$ ( $\mathrm{pH}$ 6.8), $2 \%$ SDS, $5 \%$ $\beta$-mercaptoethanol, and $8 \%$ glycerol at $95^{\circ} \mathrm{C}$. Samples were subjected to SDS-PAGE and Western blot analysis.

\section{From brain membrane fraction}

E18 rat brains were homogenized in 3 vol of buffer containing $20 \mathrm{~mm}$ HEPES, pH 7.4, $2 \mathrm{~mm}$ EDTA, 2 mм EGTA, $0.1 \mathrm{~mm}$ DTT, $100 \mathrm{~mm} \mathrm{KCl}$, $10 \mathrm{~mm} \mathrm{NaF}, 1 \mathrm{~mm}$ PMSF, 0.32 $\mathrm{m}$ sucrose, and Halt protease and phosphatase inhibitors on ice and centrifuged at $1000 \mathrm{rpm}$ for $10 \mathrm{~min}$ to remove nuclei. The postnuclear supernatant was further centrifuged at 14,000 rpm for $50 \mathrm{~min}$, and the pellet obtained was lysed in homogenization buffer containing 1\% Triton X-100 for $45 \mathrm{~min}$ on ice. This was followed by centrifugation at 12,000 rpm for 15 
A
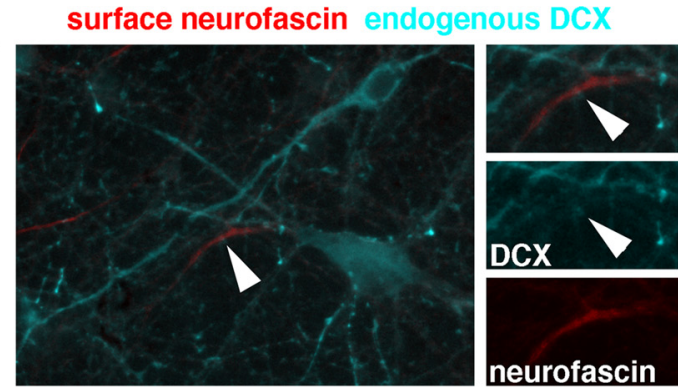

B

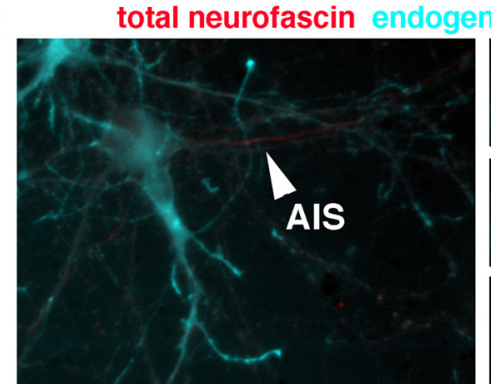

total neurofascin endogenous DCX

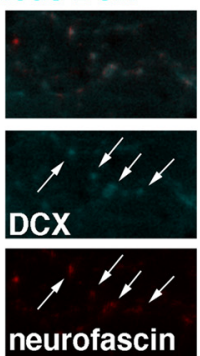

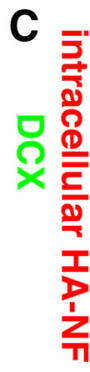

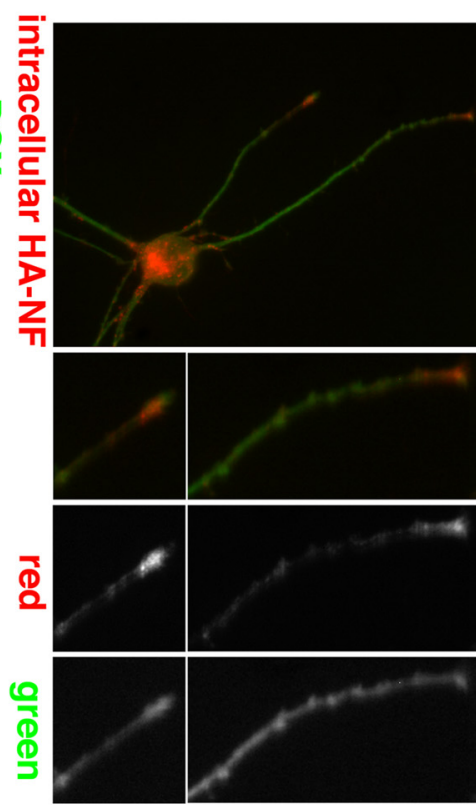

Figure 2. NF and DCX colocalize intracellularly in neurons. $\boldsymbol{A}$, Costaining of DIV9 neurons with antibodies against extracellular neurofascin (live staining; red) and endogenous DCX (aqua). No co-colocalization is observed at the AIS (arrowheads). B, Costaining of DIV9 neurons with antibodies against intracellular epitope of neurofascin (red) and endogenous DCX (aqua). No cocolocalization is observed at the AIS, but colocalizing puncta can be observed along axons and dendrites (arrows in insets). C, DIV9 neurons were transfected with HA-NF and stained with antibodies against an intracellular epitope of neurofascin in red and endogenous DCX in green. Colocalizing puncta can be observed along axons and dendrites.

min and the supernatant was used for immunoprecipitation as described above (see previous paragraph, From HEK293 cells).

\section{Polarity index}

Distal axon, AIS (ankG-positive), and dendrite fluorescence intensities were measured along a 1-pixel-wide line in ImageJ after background subtraction and normalized to length (Boiko et al., 2007). The AIS polarity of HA-neurofascin was determined by dividing average AIS intensity of HA-neurofascin surface staining by the average intensity of surface HA-neurofascin on dendrites. For endogenous NF staining, AIS intensity measurements are reported without normalizing to dendrite intensity.

\section{Detergent extractions}

Detergent extractions of live cells were carried out in BRB80 buffer ( 80 mM PIPES, 1 mм $\mathrm{MgCl}_{2}, 1$ mм EGTA, pH 6.8) or PBS++ buffer (BioWhittaker) with $0.15 \%$ Triton $\mathrm{X}-100$ at $37^{\circ} \mathrm{C}$ for $3 \mathrm{~min}$. Cells were washed once in BRB80 or PBS + + buffer and fixed.

\section{Statistical analysis}

Statistical analysis was carried out using Prism software. Datasets were first evaluated as parametric versus nonparametric. Nonparametric datasets were analyzed using Mann-Whitney $U$ test, and parametric datasets were analyzed with Student's $t$ test.

\section{Results}

\section{Downregulation of DCX affects surface distribution of NF}

Since the significance of the NF-DCX interaction was unknown, we tested whether DCX affected NF distribution in hippocampal neurons after 9-10 DIV. DCX levels were reduced by expressing short hairpin plasmids directed against rat DCX fused to GFP. One day after transfection with shDCX-GFP plasmids, endogenous DCX was partially but significantly reduced (Fig. $1 A$; red, arrow). Four different shDCX plasmids (Fig. $1 B$ ) were tested and all caused significant downregulation of DCX levels in $20 \mathrm{~h}$ compared to untransfected cells or to cells expressing the control shRandom plasmid. Average downregulation was between 30 and $40 \%$. Downregulation in a similar range after one day was previously found for electroporation of RNAi into rat embryo cortex (Bai et al., 2003). We note that the extent of downregulation of DCX in $20 \mathrm{~h}$ was variable from cell to cell. For shDCX\#2GFP, $65 \%$ of cells significantly downregulated endogenous DCX (average of $63 \%$ downregulation), whereas in the other $35 \%$ of cells expressing shDCX\#2-GFP, endogenous DCX levels were not significantly affected compared to control cells. In the remainder of this work, we used shDCX\#2-GFP.

We then tested whether the localization of endogenous neurofascin was affected by downregulation of DCX. Cultured neurons were transfected at DIV7 with either shRandom-GFP or shDCX\#2-GFP and the surface pool of endogenous NF stained before fixation at DIV14. Quantification of endogenous DCX staining revealed that only $\sim 25 \%$ of DCX on average remained after $8 \mathrm{~d}$ of shDCX expression (Fig. $1 \mathrm{~B}$ ). A small yet significant decrease in AIS intensity of NF was observed in cells expressing shDCX\#2-GFP (Fig. 1D). We then performed rescue experiments using an shDCX\#2-insensitive DCX plasmid containing seven silent point mutations. The phenotype could be partially rescued by cotransfecting resistant DCX (Fig. $1 D$ ). The AIS surface accumulation of endogenous NF could therefore be modulated by long-term decrease of DCX levels.

Since the components of the AIS, including NF, are very stable once assembled (Zonta et al., 2011), we wondered whether the small effect observed might be due to the fact that NF localization might not be dynamically modified very much once it reaches the AIS. We thus decided to determine the behavior of HA-NF (NF tagged with an extracellular HA tag) that was acutely expressed for $20 \mathrm{~h}$. This experimental paradigm allowed us to monitor newly synthesized HA-NF that was still trafficking actively. shDCX\#2-GFP or shRandom-GFP were cotransfected with HA-NF into DIV9/10 hippocampal neurons, and HA-NF surface distribution was determined by quantifying the average fluorescence intensity along dendrites and along the AIS (Fig. 1C). Since expression levels of HA-NF vary from cell to cell, the ratio of AIS 


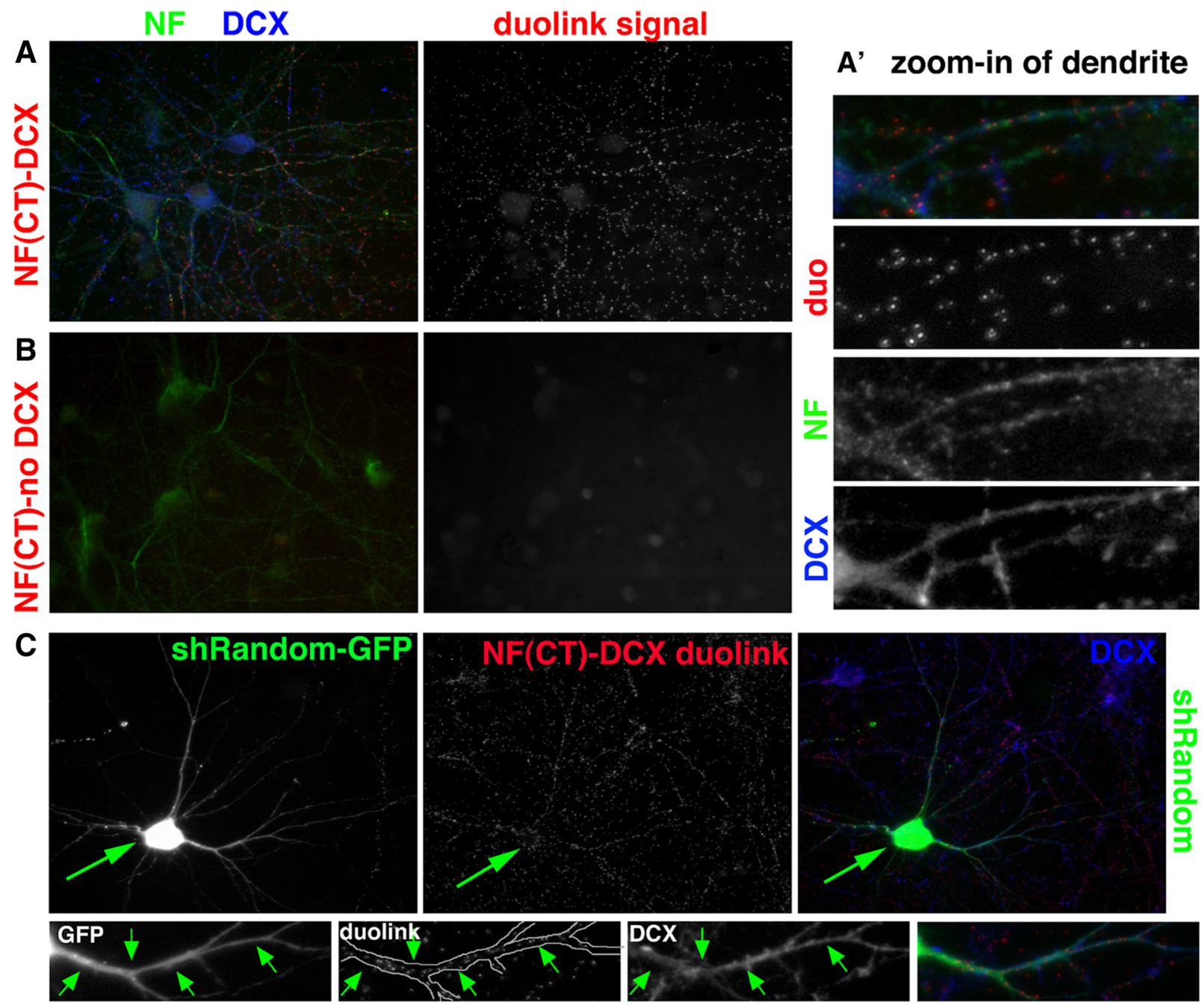

D

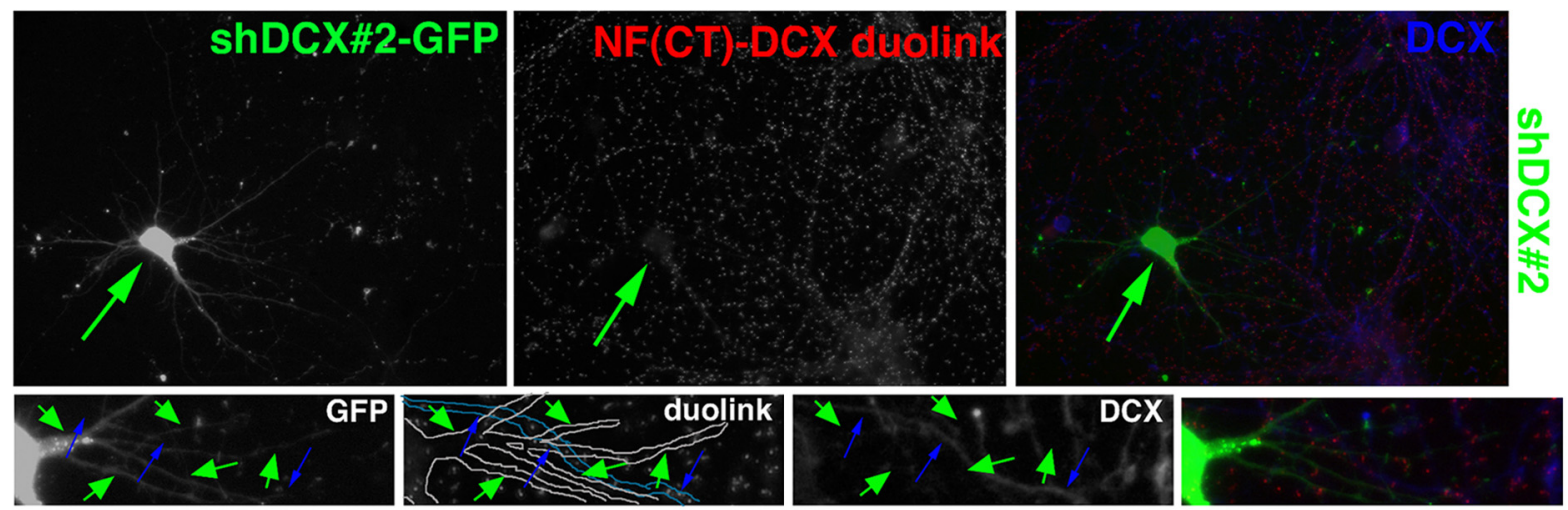

E

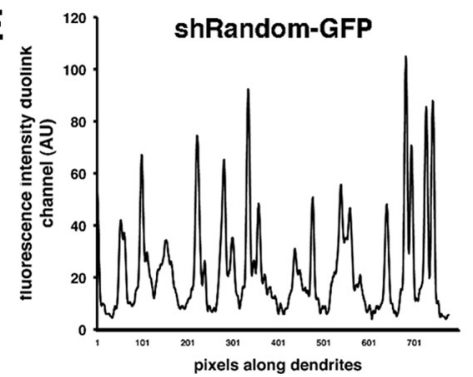

$\mathbf{F}$

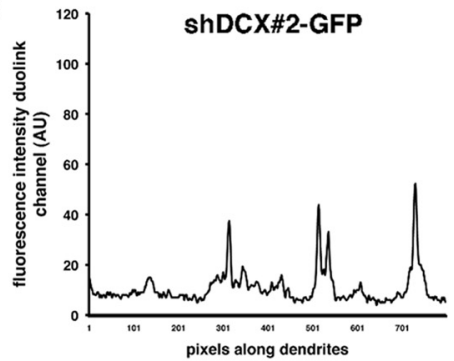

Figure 3. Neurofascin and DCX reside in close proximity in cultured neurons using Duolink. $A, B$, Duolink detection was performed on DIV12 hippocampal cultures using cytoplasmic tail-directed $\mathrm{NF}$ antibody and DCX antibody $(\boldsymbol{A})$ or only the cytoplasmic tail-directed NF antibody $(\boldsymbol{B})$, and Duolink signal was detected in red (single channel shown in right panels). Subsequently, NF antibody was detected with a green secondary, and $D C X$ antibody was detected in blue. Abundant Duolink dots were detected along dendrites in $\boldsymbol{A}$ ( $\boldsymbol{A}^{\prime}$ shows close-up of one dendrite), but no dots in the control (B). C, D, Hippocampal neurons were transfected at DIV7 with either shRandom-GFP $(\boldsymbol{C})$ or shDCX\#2-GFP (D), and Duolink was performed $5 \mathrm{~d}$ later with cytoplasmic tail-directed (Figure legend continues.) 
intensity/dendrite intensity (AIS/D polarity index) was calculated for each cell to quantify the enrichment of HA-NF on the AIS as compared to dendrites (see Materials and Methods; Boiko et al., 2007). This measure represents the relative AIS enrichment of HA-NF. The AIS/D polarity index fell in the range of 3-9 across a wide range of HA-NF expression levels, with an average AIS/D polarity index of 5-6 in control cells expressing shRandom-GFP (Fig. $1 E$; blue triangles). When shDCX\#2 was expressed, the average dendrite intensity of HA-NF on the surface was significantly increased (Fig. $1 E$; red triangles). As a result, the AIS/D polarity index was significantly decreased (Fig. 1E,F). HA-NF surface levels in the axon were not greatly affected, but a small increase in HA-NF in the distal axon was frequently observed (data not shown). Since it was difficult to unambiguously identify the distal axons of many individual cells, we focused our analysis on dendrites and AIS regions. When we tested all four independent DCX-directed shRNA constructs targeting distinct regions of the DCX transcript, the AIS/D polarity index of HA-NF was significantly reduced for all of them (data not shown). Coexpressing HA-NF, shDCX\#2, and resistant DCX-GFP in neurons for $20 \mathrm{~h}$ led to statistically significant rescue of the AIS/D polarity index of HA-NF (Fig. $1 F$ ). Short-term downregulation of DCX levels therefore led to missorting of acutely expressed HA-NF to the somatodendritic surface and concomitant decrease of the AIS/D polarity index.

We then tested the effects of shDCX on the surface distribution of other axonal receptors. Neither the axonal localization of NgCAM (chick L1; acutely expressed for $20 \mathrm{~h}$ with shDCX\#2 at DIV9/10) nor the localization of the endogenous AIS-enriched voltage-gated sodium channel (VGSC; shDCX\#2 from DIV4DIV12) was changed by expression of shDCX\#2 (Fig. 1G,H). The AIS-resident cytoskeletal ankyrinG was also not affected by downregulation of DCX for $7 \mathrm{~d}$ (Fig. 1I). Decreasing DCX levels thus led to specific mistargeting of HA-NF to dendrites, resulting in reduced relative accumulation on the AIS.

Work by others reported changes in dendritic branching patterns (primarily in young neurons) by shDCX or DCX overexpression or in DCX-/y neurons from knockout animals (Deuel et al., 2006; Cohen et al., 2008). In our cells, after $20 \mathrm{~h}$ of shDCX expression in DIV9/10 neurons, dendrite morphology was not disturbed, and even after 5-7 d of shDCX expression (starting at DIV 7), the extent of the dendritic arbor was still extensive (data not shown).

\section{DCX colocalizes with an intracellular pool of NF.}

To determine where in neuronal cells DCX-NF interactions might be taking place, we stained DCX and surface NF (using an antibody directed against an extracellular epitope; Fig. 2A) in cultured hippocampal neurons and compared them to the localization of total NF (using an antibody against the cytoplasmic tail; Fig. 2B). Surface NF (red) was highly enriched on the AIS (arrowhead), as described previously (Hedstrom and Rasband,

\footnotetext{
$\leftarrow$

(Figure legend continued.) NF antibody and DCX antibody (as in A). C, Abundant Duolink signal (middle panel) was detected along transfected (green) dendrites, which expressed DCX (blue). Single channels of one transfected dendrite (outlined and marked by green arrows) are shown in the small panels below. D, Few Duolink dots are present on the sh-DCX\#2-GFP transfected neuron (green arrow), but abundant Duolink dots are present along dendrites of nontransfected cells, still expressing $D C X$ (blue). Single channels of one transfected dendrite (outlined by green arrows) are shown in the small panels. A nontransfected dendrite crossing the field is outlined with blue and marked with blue arrows and still expresses DCX. Line scans of the Duolink signal along the dendrites corresponding to the small panels are shown in $\boldsymbol{E}$ (shRandom-GFP) and $\boldsymbol{F}$ (shDCX\#2-GFP).
}

2006; Boiko et al., 2007; Dzhashiashvili et al., 2007). In agreement with previous data (Schaar et al., 2004; Tint et al., 2009), DCX was found throughout the cell, often enriched in the distal tips of axonal and dendritic processes (aqua). Not surprisingly then, surface NF and DCX were not strikingly colocalized (Fig. $2 A$ ). In contrast, intracellular NF partially colocalized with DCX in apparent clusters, particularly in dendrites and at dendrite tips (Fig. $2 \mathrm{~B}$; arrows). A similar colocalization in clustered puncta could also be observed when HA-NF (red, detected with an anticytoplasmic tail antibody) was expressed exogenously in cultured neurons (Fig. 2C). There was thus a readily detectable intracellular pool of NF, which partially colocalized with DCX. This intracellular pool of NF was widely dispersed throughout the whole cell and did not cluster underneath the AIS.

Next, we determined whether DCX and NF resided in close enough proximity in neurons to give a positive Duolink signal ( $\sim 40 \mathrm{~nm}$ according to the manufacturer; see Materials and Methods). Duolink was performed on DIV10-12 neurons using a mouse anti-neurofascin cytoplasmic tail (CT)-directed antibody and a rabbit anti-DCX antibody (Fig. $2 B$ ). Multiple bright dots suggestive of closely apposed binding of the two antibodies were observed (red channel in Fig. $3 A, A^{\prime}$ ). The positive Duolink signal was particularly apparent in the major dendrites but could also be observed in the soma and less abundantly in axons. The AIS itself was not enriched in labeling (Fig. $3 A$ ). Importantly, no dots were observed if one of the primary antibodies was omitted from the staining procedure (Fig. $3 B$ ). To further verify the specificity of the Duolink signal, we depleted DCX in neurons with shDCX\#2-GFP. When shDCX\#2 plasmid was expressed in neurons for $5 \mathrm{~d}$ (fixed DIV12), the specific labeling efficiency of Duolink between NF and DCX was substantially reduced (red channel in Fig. 3D) compared to cells expressing a control shRandom plasmid (Fig. 3C). Dendrites of cells expressing shDCX\#2 (green, Fig. 3D) had little to no DCX staining (blue) and few Duolink dots (red) associated with the transfected (green) dendrites. In contrast, shRandom-expressing neurons displayed Duolink signals indistinguishable from untreated cells (Fig. 3C). A close-up of dendrites expressing shRandom or shDCX\#2 is shown in the small panels. Green arrows indicate dendrites expressing the shRNA constructs (Fig. 3C,D, small panels) and were mostly devoid of DCX and Duolink signal. Blue arrows indicate the dendrite of a nontransfected neuron still showing DCX (blue) and numerous Duolink dots (Fig. 3D, small panels; red). Line scans of the green dendrites shown in the small panels illustrate the density of red Duolink dots for easier comparison (Fig. $3 E, F)$. Thus specific Duolink labeling between endogenous NF and DCX could be observed, indicating close proximity of the endogenous proteins in dendrites and axons.

\section{Phospho-NF capable of binding DCX is present in hippocampal cultures.}

To confirm previous findings that NF was in a complex with a membrane-bound fraction of DCX (Kizhatil et al., 2002), we performed immunoprecipitations from brain membrane fractions using anti-NF antibody and probing the immunoblot for DCX (Fig. 4A). A coimmunoprecipitating band was observed (lane 2) that was not present when nonimmune antibody (lane 1) was used as a control. A membrane fraction from brain therefore contains both NF and DCX, and the two proteins can be immunoisolated as a complex from the membrane fraction.

To test whether phosphoFIGQY-NF (the phospho-form capable of binding DCX) was present in hippocampal cultures, we immunoprecipitated with a pan-NF antibody and probed with a 


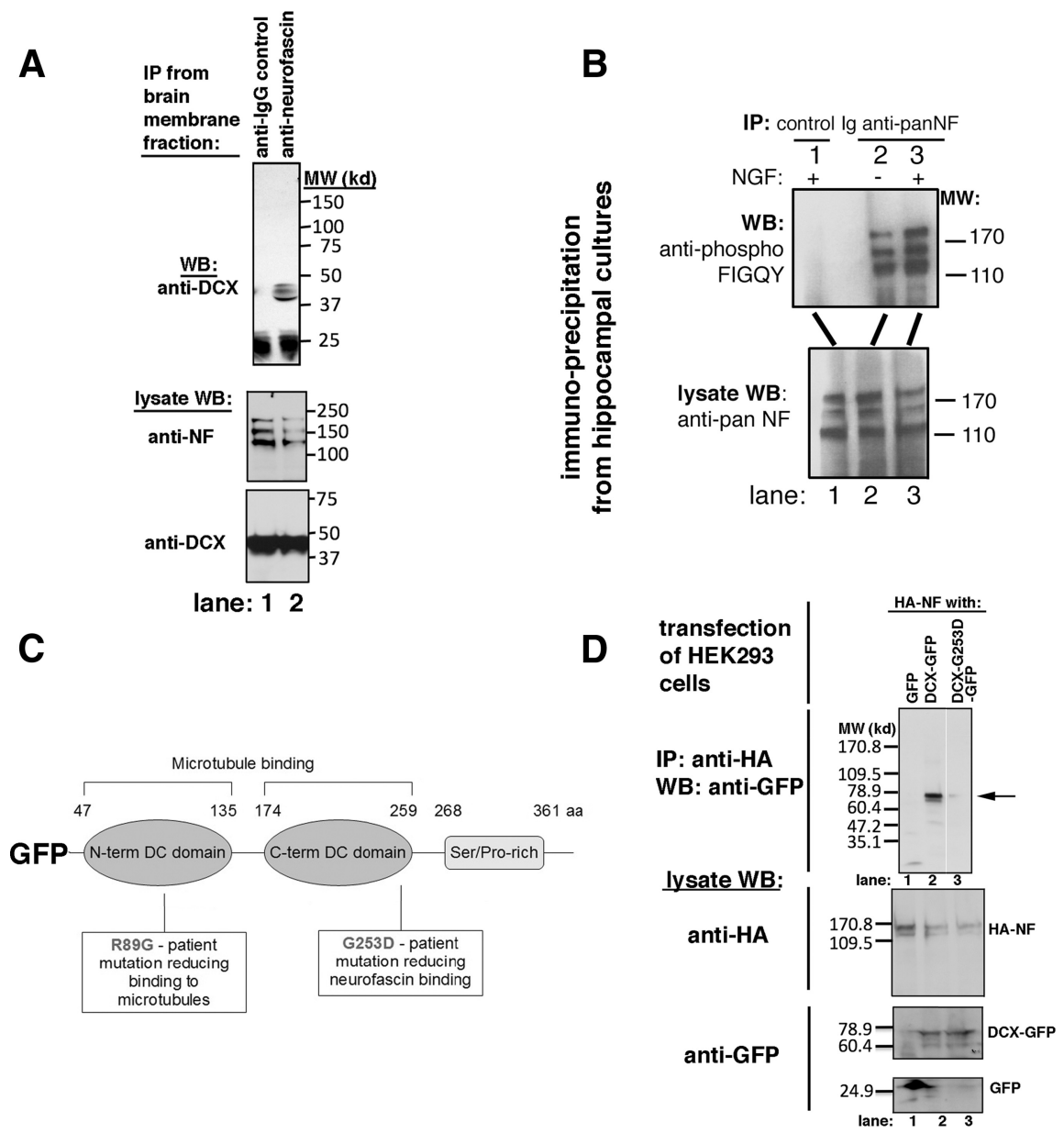

Figure 4. Neurofascin/DCX complex in vitro. $\boldsymbol{A}$, Coimmunoprecipitation of $D C X$ and neurofascin from $E 18$ rat brain membrane fraction with nonimmune IgG (lane 1) or anti-neurofascin antibody (lane 2). The levels of neurofascin and DCX in the lysates were determined by Western blotting (WB). IP, Immunoprecipitation $\boldsymbol{B}$, Presence of phospho-FIGQY neurofascin in hippocampal cultures. Hippocampal cultures at DIV10 were treated with $100 \mathrm{ng} / \mathrm{mI} \mathrm{NGF} \mathrm{for} 48 \mathrm{~h}$ (lanes 1 and 3) or not (lane 2), and membrane fractions were prepared and used for immunoprecipitation with control lgG (lane 1) or anti-neurofascin (NF) antibody (lanes 2 and 3), and the immunoprecipitates were probed with anti-phosphoFIGQY antibody. PhosphoFIGQY could be detected even without additional NGF, but NGF treatment increased the levels of phosphoFIGQY. MW, Molecular weight. C, Diagram of DCX-GFP indicating the DC repeats and the positions of the G253 and R89 patient mutations. D, HEK293 cells were transfected with HA-NF and GFP (lane 1), DCX-GFP (lane 2), or DCX-G253D-GFP (lane 3). Lysates were prepared and used for WB against HA or GFP or used for IP with anti-HA antibody. DCX-GFP was efficiently immunoprecipitated, whereas DCX-G253D-GFP was not (arrow).

phosphoFIGQY-specific antibody (Kizhatil et al., 2002). Three prominent NF isoforms were detected (likely corresponding to different splice isoforms) (Fig. 4B, lane 2).

Treating 10 DIV cultures with $100 \mathrm{ng} / \mathrm{ml}$ NGF for $2 \mathrm{~d}$ increased the abundance of phosphoFIGQY-NF. The slowest mobility band, likely NF-186, showed a 2.7-fold increase in phosphoFIGQY reactivity after NGF treatment (Fig. $4 B$, compare lanes 2 and 3). PhosphoFIGQY reactivity was thus easily detected in untreated cultures (lane 2), likely due to the presence of growth factors in the medium, and levels were further increased by NGF.

Expression of DCX mutants deficient for NF binding interfere with HA-NF enrichment on the AIS.

Since reduction of DCX levels decreased the relative AIS enrichment of HA-NF (Fig. 1), we wondered whether expression of a DCX mutant that no longer bound NF affected the relative AIS enrichment of HA-NF. We therefore created a point mutant in
DCX previously shown to weaken the interaction of DCX with neurofascin fivefold (Kizhatil et al., 2002; patient mutation DCX-G253D; Fig. 4C) and tested its ability to coimmunoprecipitate with HA-NF. Wild-type DCX-GFP coimmunoprecipitated with HA-NF from HEK293 cells (Fig. 4D, lane 2, arrow). In contrast, coimmunoprecipitation of DCXG253D-GFP with HA-NF was greatly reduced (Fig. $4 D$, lane 3 ).

When DCX-G253D-GFP was expressed with HA-NF in neurons, the relative AIS enrichment of HA-NF was decreased compared to GFP as control (Fig. $5 B, C, E, F$ ). The decrease was due to a significant increase in average somatodendritic fluorescence of surface HA-NF (Fig. 5B). Coexpression of wild-type DCX-GFP, on the other hand, did not change the relative AIS enrichment of HA-NF (Fig. 5A, $C, D, F$ ). The patient mutation DCX-G253D, therefore, acted in a dominant-negative manner to mislocalize HA-NF.

We then tried to analyze the localization of endogenous NF in DIV10-14 cells expressing WT-DCX-GFP, DCX-G253DGFP, or GFP. We did not observe any effects of expressing any of the constructs for $20 \mathrm{~h}$ on endogenous NF localization, consistent with the slow turnover of NF at the AIS. When we expressed the DCXG253D for longer periods of time (7 d), we started to see changes in the morphology of neurons expressing DCX-G253D, indicative of poor health. Endogenous NF localization could therefore not be reliably analyzed.

\section{DCX-G253D is still bound along microtubules in cells.}

Since the best-characterized cellular activity of DCX is microtubule binding and bundling (Liu, 2011), we asked whether DCX-G253D-GFP still bound to microtubules in cells or whether the HA-NF mislocalization phenotype correlated with lack of microtubule binding activity. For this purpose, we used a previously established "in-cell" binding assay in which live cells were extracted in $0.15 \%$ Triton X-100 in microtubule-stabilizing buffer (BRB80) before fixation (Sapir et al., 2000). The ability of DCX to remain associated with microtubules after live detergent extraction was then determined by immunostaining. MAP2 was used to counterstain dendritic microtubules (red). In agreement with previous work, DCX-GFP (Fig. $6 A^{\prime}$ ) remained detergent-resistant, presumably by binding to microtubules. We then performed the same extractions on neurons expressing DCX-G253D-GFP (Fig. 6B, $B^{\prime}$ ). DCX-G253D-GFP could be detected brightly in neurons without extraction (Fig. $6 B$ ) or after extraction in BRB80 (Fig. 6 $B^{\prime}$ ). Surprisingly, DCX and DCXG253D were more resistant to extractions than MAP2 itself, whose staining was frequently diminished.

To demonstrate that detergent-resistant DCX remained bound to microtubules rather than other insoluble cytoskeletal 
components, we repeated the extractions in COS7 cells where individual microtubules could be easily resolved. We first validated the detergent extractions in COS7 cells and found that microtubules (blue) and $\mathrm{F}$-actin (red) remained intact after detergent extraction in BRB80 (Fig. 6C). To confirm that detergent resistance was attributable to microtubule binding rather than microfilament binding, we used a microtubule-destabilizing buffer $\left(\mathrm{PBS}^{++}\right)$for detergent extractions in which microtubules (blue) were not stably maintained and thus lost, whereas microfilaments (F-actin, red) remained intact (Fig. 6D). When DCX-GFP was expressed in COS7 cells and then extracted in BRB80, detergent-resistant DCX-GFP colocalized with splayed individual microtubules (Fig. 6Ea). In addition, DCXGFP induced thick microtubule bundles (Fig. 6Eb) in the highly expressing cells, consistent with previous observations (Sapir et al., 2000). When extractions were performed in $\mathrm{PBS}^{++}$, DCX-GFP was not detergent resistant, nor were microtubules (Fig. 6F). To identify transfected cells, plasmids encoding lamin-cherry (red) were cotransfected because lamin is detergent resistant, even in $\mathrm{PBS}^{++}$. Since the cotransfection rate of two different plasmids in COS7 cells was higher than $80 \%$ (data not shown), most of the lamincherry expressing cells were also transfected with DCX-GFP, but lost DCX-GFP during the extractions. Next we tested DCX-G253D in the same assay. We found that DCX-G253D-GFP still decorated microtubules and induced thick bundles (Fig. 6G). Therefore, DCX-G253D-GFP had not lost microtubule binding or bundling capacity. We note that the detergent extraction assay measures overall microtubule binding but does not assess more subtle changes in the interaction and function of mutant DCX with microtubules. The same caveat exists for in-bulk microtubule binding assays (Sapir et al., 2000), which are carried out entirely in vitro with purified proteins. These observations raised the possibility that the HA-NF localization defect caused by expression of DCX-G253D-GFP was because it could not bind NF and not because of a defect in microtubule binding.

\section{Overexpression of a microtubule-binding deficient DCX} (DCX-R89G) did not cause mislocalization of HA-NF.

The NF-localizing activity of DCX could be disrupted by a NF binding-deficient DCX mutant (see above, Expression of DCX mutants deficient for NF binding interfere with HA-NF enrichment on the AIS), but microtubule binding might be additionally needed. We therefore tested whether a DCX mutant incapable of binding microtubules phenocopied the DCX-G253D phenotype with respect to HA-NF distribution. We predicted that it would only phenocopy if the NF-localizing activity of DCX was dependent on its microtubule-binding activity in addition to its
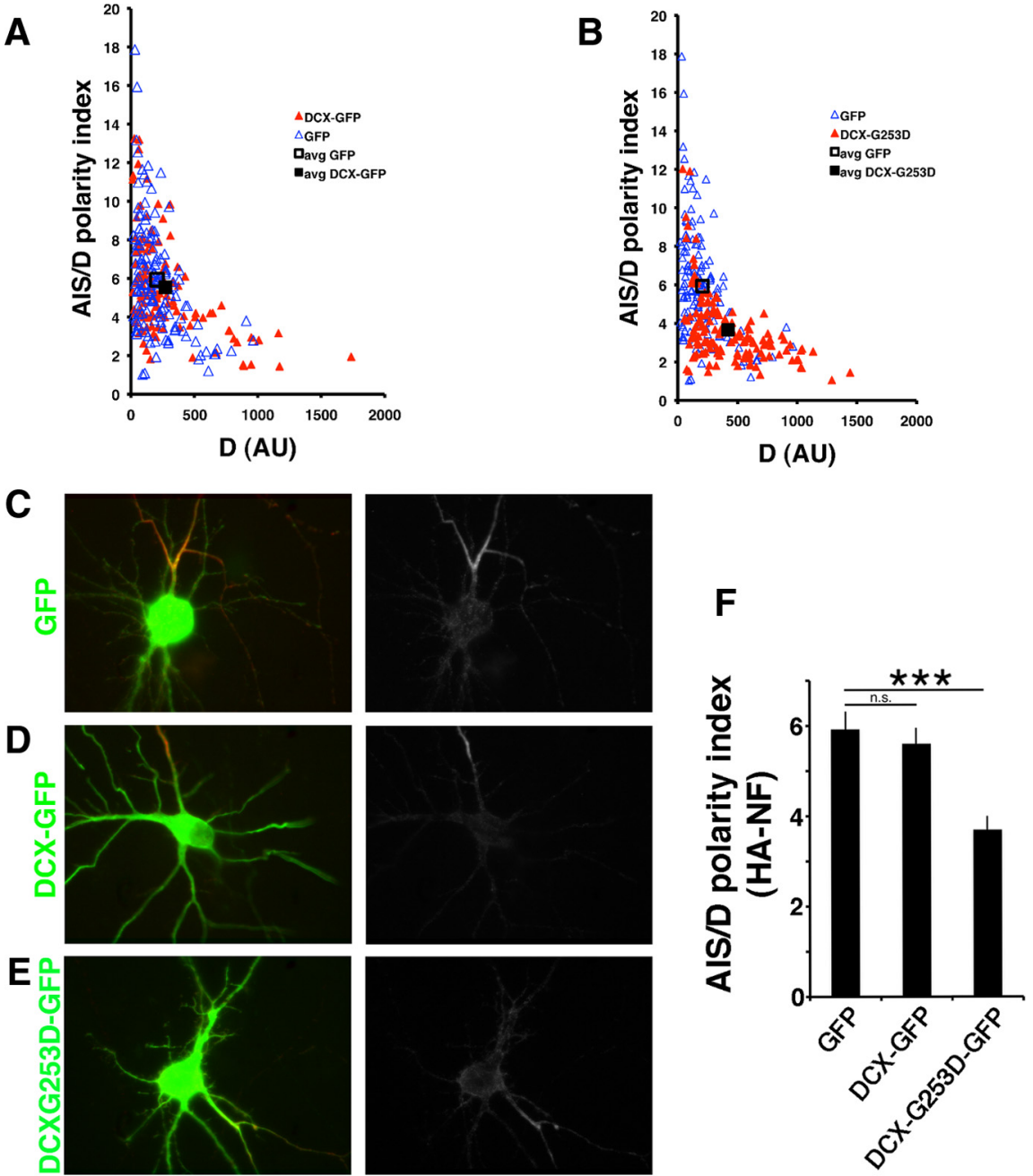

Figure 5. Coexpression of DCX mutants deficient for NF binding interferes with HA-NF surface distribution. $A, B, S$ catter plots of dendrite intensity and AIS/D polarity index for individual cells transfected with GFP (blue) or DCX-GFP (red) (B) for $20 \mathrm{~h}$, or with GFP ) change AIS/D polarity index. DCX-G253D-GFP decreases relative AIS targeting of HA-NF. N = 139 cells for GFP, 119 cells for DCX-GFP, and 136 cells for DCX-G253D from three independent experiments. ${ }^{* * *} p<0.0001$; Bars, SEM.

NF-binding activity. If DCX played a role in localizing NF independently of its microtubule-binding activity, a microtubulebinding deficient DCX would not phenocopy DCX-G235D. We therefore generated DCX-R89G-GFP, a previously described patient mutation reported to be incapable of binding microtubules (Kim et al., 2003; Fig. 4C), and tested the ability of DCX-R89G-GFP to bind microtubules. We note that DCX-R89G-GFP accumulated at lower levels in non-neuronal cells than WT DCX-GFP (Fig. 7A). In cells transfected with DCX-R89G-GFP and stained without detergent extraction, DCX-R89G-GFP was diffuse in the cytoplasm and did not colocalize along microtubules (Fig. 7C). When cells were detergent extracted live in BRB80, DCX-GFP (green) decorated microtubules (red) and caused bundling (Fig. 7D), whereas DCX-R89G-GFP was not detergent resistant and no staining remained, even though microtubules are stabilized (blue) (Fig. 7E). Lamin-cherry was used again to identify transfected cells. These experiments confirmed that DCX-R89G was deficient for microtubule-binding.

We then cotransfected DCX-R89G-GFP and HA-NF into hippocampal neurons and determined the AIS/D polarity of surface 

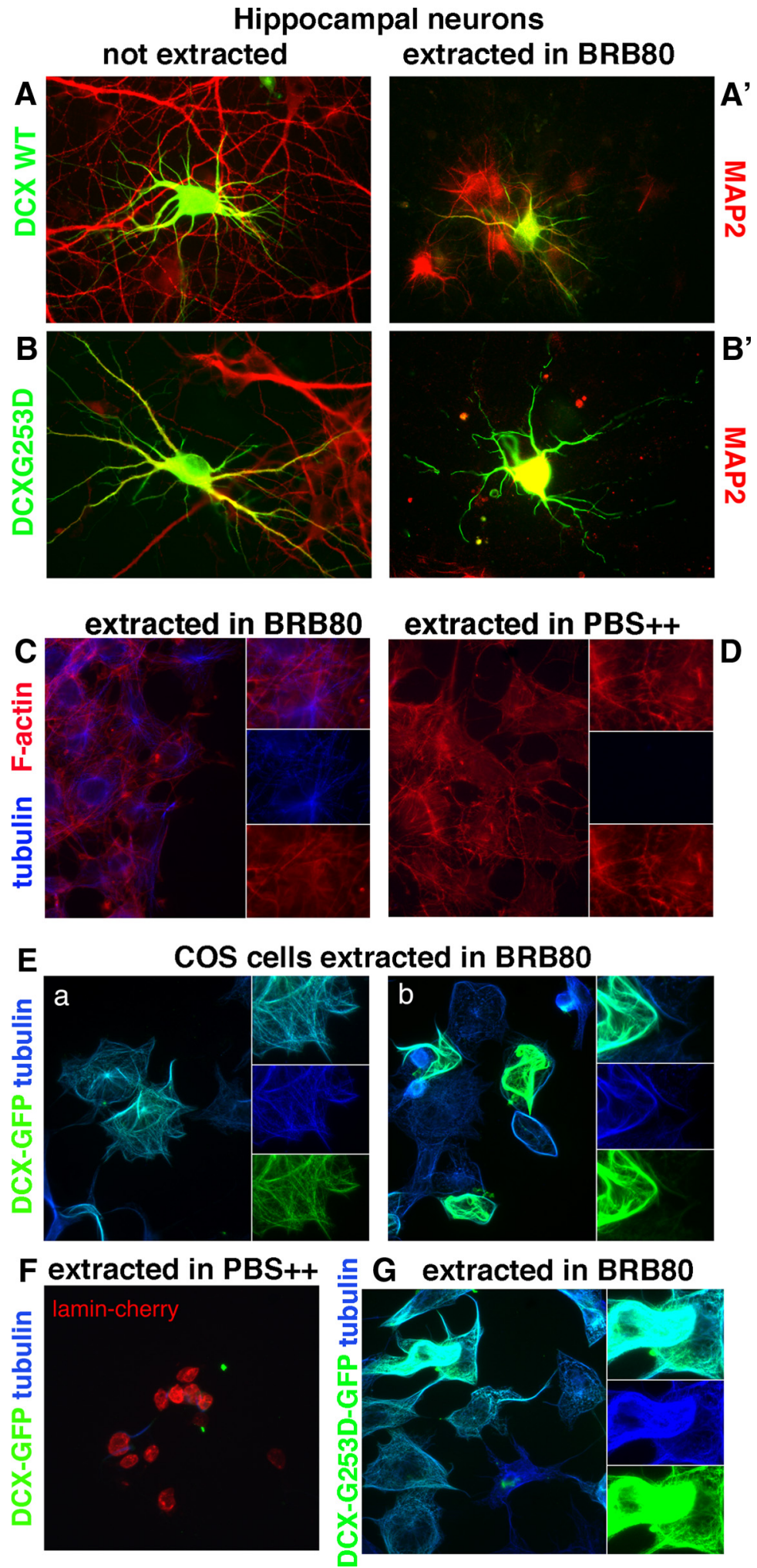

Figure 6. DCX-G253D mutant still binds microtubules in cells. $A-B^{\prime}$, Neuronal cultures were transfected with DCX-GFP $\left(A, A^{\prime}\right)$ or DCX-G253D$\operatorname{GFP}\left(\boldsymbol{B}, \boldsymbol{B}^{\prime}\right)$.After20h, livecultures wereeither fixed $(\boldsymbol{A}, \boldsymbol{B})$ ordetergentextracted in microtubule-stabilizing buffer BRB80 before fixation $\left(\boldsymbol{A}^{\prime}, \boldsymbol{B}^{\prime}\right)$. The transfected proteins were detected with anti-GFP (green) antibodies and counterstained for MAP2 (red). Both constructs expressed well in transfected neurons without extractions. If cells were extracted live before fixation, both DCX constructs were still easily detected on detergent-insoluble cytoskeleton. C, COS cells extracted in BRB80 and 0.15\% Triton X-100 before fixation show intact microtubule (blue) and microfilament (red) networks. $D$, COS cells extracted in PBS ${ }^{++}$and $0.15 \%$ Triton X-100 before fixation show intact microfilament network (red), but loss of microtubules (blue). $\boldsymbol{E}-\mathbf{G}$, COS cells were transfected with DCX-GFP $(\boldsymbol{E}, F)$ or DCX-G253D-GFP (G) and extracted in BRB80 (E, G) or microtubule-destabilizing buffer $\mathrm{PBS}^{++}(\boldsymbol{F})$. DCX-GFP decorates individual microtubules (blue) (Ea) and induces microtubule bundles when highly expressed (Eb). After extraction in PBS ${ }^{++}$, DCX is nondetectable in transfected cells (expressing lamin-cherry) (F). DCX-G253D-GFP also associates with microtubules and still induces bundles (G).
HA-NF 20 h later. In contrast to DCXG253D-GFP, DCX-R89G-GFP did not impair localization of HA-NF to the AIS (Fig. 7F). Lastly, we tested whether DCXR89G-GFP was still able to bind HA-NF by coimmunoprecipitation from transfected HEK293 cells. HA-NF was still coimmunoprecipitated with DCX-R89GGFP (Fig. 7B, lane 3). As expected from the much lower expression levels of DCXR89G-GFP (Fig. 7A), less DCX-R89G-GFP was detected in the immunoprecipitate.

HA-NF undergoes endocytosis in hippocampal neurons

Next, we tested whether endocytosis might be involved in the relative accumulation of neurofascin at the AIS. This idea arose because shDCX and DCX-G253D both caused abnormal accumulation of HA-NF on dendrites, suggesting that normally HA-NF might be removed from these places by endocytosis. First, we tested whether NF underwent endocytosis in neurons. Endocytosis of L1, a close family member of NF, is well documented, but endocytosis of NF has not been described. In fact, rat NF lacks the endocytosis motif present in the cytoplasmic tail of L1 (Hortsch, 2000). Using anti-HA antibody feeding of live neurons expressing HA-NF, endocytosis could be readily detected in about $30 \%$ of transfected neurons, with somatodendritic puncta being the most prominent (Fig. 8A; red). A significant pool of HA-NF remained stably assembled at the AIS (Fig. 8A; green) and did not endocytose within $30 \mathrm{~min}$. To ascertain that the observed puncta corresponded to endosomes, we labeled additionally with several markers for different subsets of endosomes, including early endosomes (EEA1-positive), recycling endosomes (rab11-positive), and late endosomes/ lysosomes (LAMP2-positive). Internalized HA-NF colocalized extensively with EEA1 (Fig. $8 B$ ) and partially with rab11-GFP (Fig. $8 C$ ). Little if any colocalization was observed with LAMP2 after a 90 min chase (Fig. 8D).

To determine whether endogenous NF could also undergo endocytosis, we carried out endocytosis experiments using $\mathrm{NF}$ antibodies. In young neurons (DIV3), endocytosis of NF could be observed in dendrites, soma, and axons (Fig. $8 E$; red). Simultaneous uptake of L1-antibodies was carried out as a positive control. Endocytosed L1 (green) could be detected readily alongside with endocytosed NF (red). NF thus undergoes endocytosis in young neurons. After DIV5, endocytosis of NF became more difficult to detect and less frequent (data not shown). In mature 
neurons (DIV10 and older), endocytosis of endogenous NF was not observed, indicating that assembled NF is stable at the AIS. Since acutely expressed HA-NF in mature neurons still endocytosed (Fig. $8 A$ ), we postulate that NF endocytoses most vigorously during its biosynthetic delivery and transport, but is stable once it reaches the AIS.

To test whether endocytosis contributed to AIS localization of NF, we inhibited clathrin-mediated endocytosis in neurons by expressing a dominant-negative Eps 15 mutant, E $\Delta 95 / 295-G F P$, which titrates out AP2. An inactive Eps15 mutant lacking the AP2 binding site, Eps15-DIII $\Delta 2-G F P$ (Benmerah et al., 1999), was used as a control. The efficacy of endocytosis inhibition by E $\Delta 95 / 295-G F P$ was confirmed by using transferrin uptake in neurons (data not shown). Coexpressing the inactive Eps15 mutant Eps15-DIII $\Delta 2$-GFP showed normal AIS/D PI values of HA-NF of $>6$. In contrast, coexpression of the dominantnegative mutant E $\Delta 95 / 295-$ GFP significantly decreased AIS/D PI values for HA-NF (Fig. $8 F$ ). The effect on endogenous NF could not be determined due to increasing toxicity of the dominantnegative protein after expression for several days. These results suggested that endocytosis contributed to the precise AIS accumulation of NF.

Endocytosis of HA-NF is stimulated by coexpression of DCX-GFP in PC12 cells Since both inhibition of endocytosis and downregulation of DCX resulted in missorting of HA-NF to dendrites, we wondered whether DCX might be directly involved in HA-NF endocytosis. To test this, we carried out endocytosis experiments in PC12 cells, which do not express DCX endogenously. PC12 cells were transfected with HA-NF, either with or without DCXGFP, and anti-HA endocytosis assays were carried out 20 h later. HA-NF endocytosis in control cells without DCX-GFP was mostly low (Fig. 9A, top row). In cells expressing DCX-GFP and HA-NF, many cells ( $>50 \%)$ showed brightly stained endosomes containing anti-HA antibodies (Fig. 9A, bottom row). The extent of endocytosis was then quantified using ImageJ (Yap et al., 2010). DCX-GFP cells showed significantly increased HA-NF endocytosis on average (Fig. 9B) compared to control cells not expressing DCX-GFP. DCX was therefore able to increase the endocytosis of HA-NF in PC12 cells. We note that the range of NF endocytosis levels was large, suggesting an apparently nonresponsive population of transfected cells $(\sim 30 \%)$ that did not show increased levels of HA-NF endocytosis upon DCX overexpression, and a population of highly responsive cells that increased NF endocytosis dramatically. The basis for this variability is currently

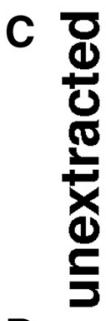

$\mathbf{F}$
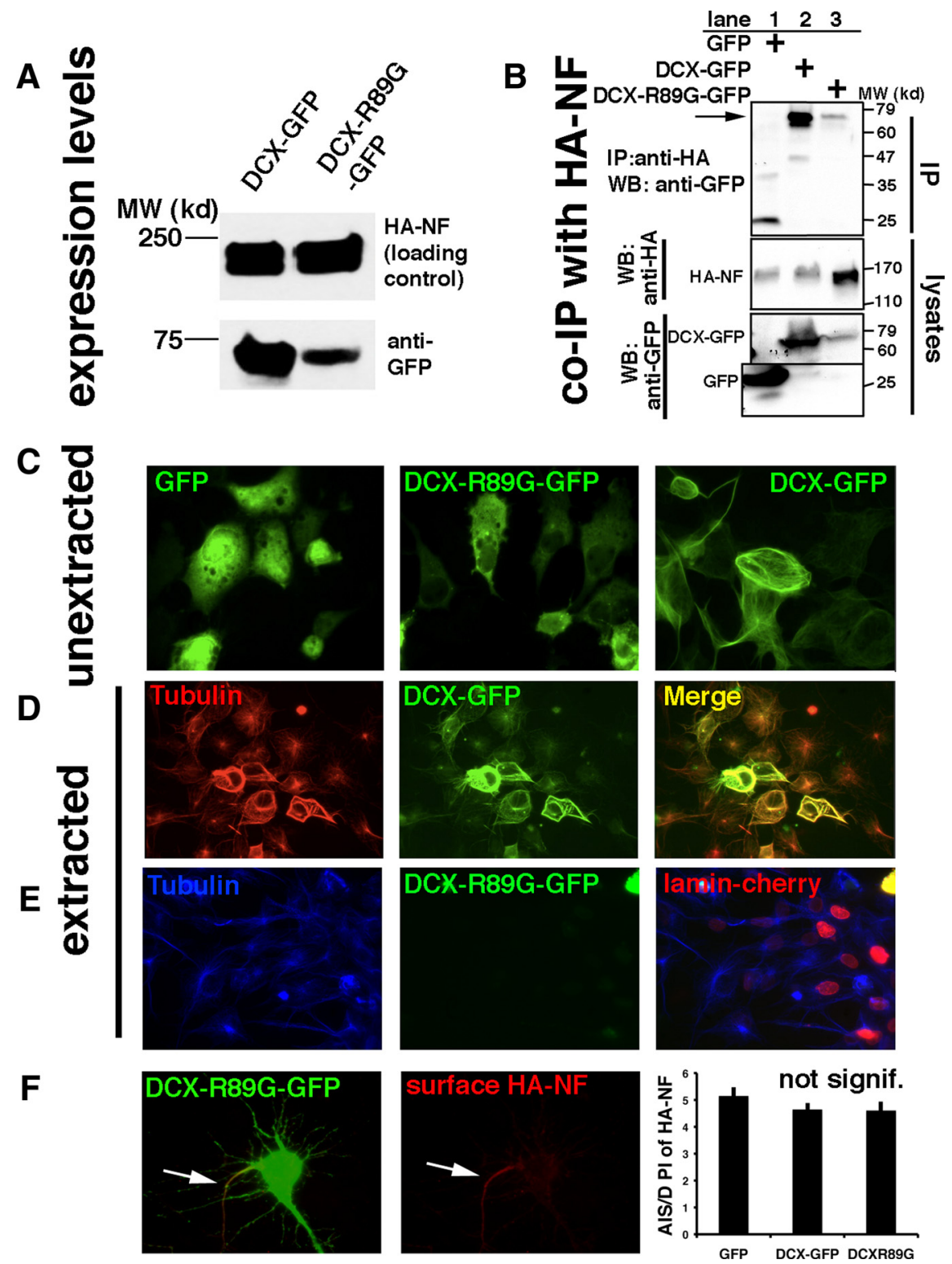

Figure 7. Overexpression of a microtubule-binding deficient DCX (DCX-R89G) does not cause mislocalization of HA-NF. A, Expression levels of DCX-R89G-GFP were determined in lysates of HEK293 cells coexpressing HA-NF and either DCX-GFP or DCXR89G-GFP. HA-NF was used for normalization of transfection efficiency and loading. MW, Molecular weight. $\boldsymbol{B}$, HEK293 cells were transfected with HA-NF and GFP (lane 1), DCX-GFP (lane 2), or DCX-R89G-GFP (lane 3). Lysates were prepared and used for Western blotting (WB) against HA or GFP or used for immunoprecipitation (IP) with anti-HA antibody. DCX-GFP was efficiently immunoprecipitated (top panel, arrow). DCX-R89G-GFP was expressed at much reduced levels in the lysate, but could still be detected as a coimmunoprecipitating (co-IP) band (lane 3; arrow). C-E, DCX-R89G-GFP has reduced microtubule-binding capacity. C, COS cells were transfected with GFP, DCX-R89G-GFP, or DCX-GFP, fixed, and stained with anti-GFP antibody. GFP and DCX-R89G-GFP are cytosolic and are not associated with microtubules, whereas DCX-GFP decorates microtubules. $\boldsymbol{D}, \boldsymbol{E}$, COS cells transfected with DCX-GFP (D) or DCX-R89G-GFP (E) were extracted in BRB80. DCX-GFP (green) localizes along microtubules (red) (D). DCX-R89GGFP, on the other hand, is not detectable (green) despite the presence of microtubules (blue) (E). Lamin-cherry was cotransfected (red) with DCX-R89G-GFP to mark transfected cells. $\boldsymbol{F}$, Expression of DCX-R89G-GFP in hippocampal neurons does not lead to mislocalization of surface HA-NF (red). A representative experiment is shown (of three independent experiments). $N=63$ cells for GFP, 57 cells for DCX-GFP, and 77 cells for DCX-R89G-GFP. Bars, SEM. The differences are not statistically significant.

not known, but it might be due to phosphorylation regulation of DCX itself.

If the increase in endocytosis observed with DCX was due to a direct interaction with HA-NF, the NF binding-deficient mutant DCX-G253D should not be able to boost HA-NF endocytosis in PC1 2 cells. Indeed, DCX-G253D-GFP did not increase HA-NF endocytosis compared to WT-DCX (Fig. 9B). Surface expression of HA-NF was quantified independently and was the same for 


\section{A exogenous}

surface HA-NF endocytosed HA-NF

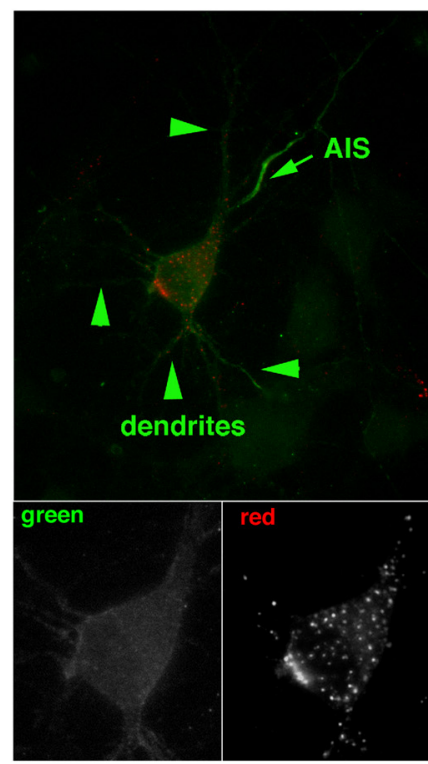

\section{E}

\section{endogenous} endocytosed NF endocytosed L1

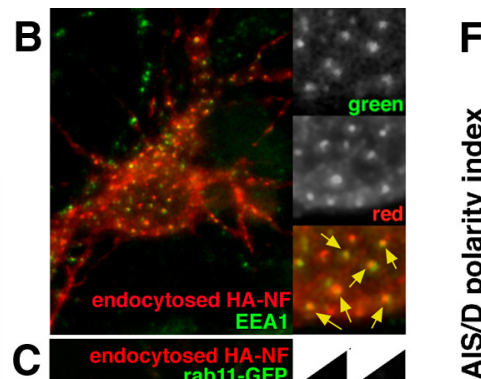

F HA-NF

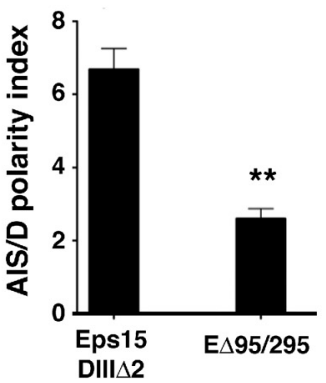

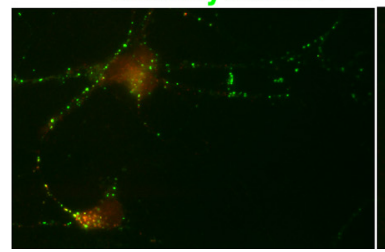
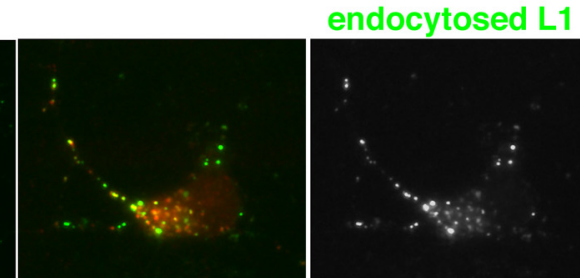

endocytosed NF
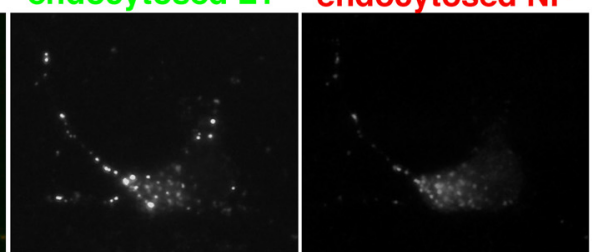

Figure 8. Neurofascin undergoes endocytosis in hippocampal neurons. A, HA-NF can be found in endosomes (red), especially in the somatodendritic domain after 30 min of rabbit anti-HA antibody uptake. Surface HA-NF (green) was stained with mouse anti-HA antibody before fixation and permeabilization. A zoom image of the soma region is shown in the bottom panels, with each channel shown separately. $\boldsymbol{B}-\boldsymbol{D}$, Costaining of endocytosed HA-NF (red) with markers (green) to early endosomes (EEA1) (B), recycling endosomes (rab11-GFP) ( $\boldsymbol{C}$, and late endosomes/lysosomes (LAMP2) after 90 minute chase $\boldsymbol{D}$, Zoomed-in regions are shown as single channels in the small panels. Yellow arrows point at colocalizing puncta. $\boldsymbol{E}$, DIV 3 hippocampal neurons were incubated live with antibodies against extracellular domains of NF (red) and L1 (green) for $30 \mathrm{~min}$. Both L1 and NF can be detected in endosomes in the soma as well as along processes. One cell is shown with single channels. $F$, Dominant-negative Eps15 construct (E $\Delta$ 95/295-GFP) and an inactive Eps15 construct (Eps15DIII $\Delta 2$-GFP) were expressed together with HA-NF for 20 h, and AIS/D polarity index was determined. Inhibition of clathrin-mediated endocytosis with E $\Delta 95 / 295$-GFP decreased AIS localization compared to controls (Eps15DIIID2-GFP). $N=15-30$ cells per experiment and condition in three independent experiments. ${ }^{* *} p<0.001 ;$ Bars, SEM.

DCX-GFP or DCX-G253D-GFP expressing cells (data not shown). We then tested whether the microtubule bindingdeficient DCX, DCX-R89G, still promoted HA-NF endocytosis, or whether microtubule binding was required for this activity. HA-NF endocytosis was increased significantly by expressing DCX-R89G-GFP in PC12 cells, similar to wild-type DCX-GFP (Fig. 9C). Thus, to stimulate NF endocytosis, NF-binding but not microtubule-binding activity of DCX was required.

To determine whether DCX-GFP boosted the internalization of a wide range of cargo molecules or whether this effect was selective for HA-NF, we also performed endocytosis assays of L1-myc and transferrin in PC12 cells. Neither L1-myc (Fig. 9D) nor transferrin endocytosis (Fig. 9E) were significantly increased by the coexpression of DCX-GFP.

Endocytosis of endogenous NF is diminished after downregulation of DCX in neurons

Lastly, we tested whether downregulation of DCX would impact the ability of NF to endocytose in young neurons. NF endocytosis assays were carried out in DIV3 hippocampal neurons plated after electroporation with shRandom-GFP (Fig. 10A) or shDCX\#2-GFP (Fig. 10 B). Cultures were stained with anti-GFP (green), anti-DCX (blue), and anti-mouse secondary antibodies to detect endocytosed NF (red) (Fig. 10A,B). Endocytosis was quantified in transfected neurons (arrow) and compared to untransfected neurons in the same field. The average NF endocytosis levels were significantly reduced in neurons expressing shDCX\#2-GFP (Fig. 10C) compared to untransfected cells or neurons expressing shRandom-GFP. Many untransfected neurons $(\sim 40 \%)$ that expressed DCX also showed low levels of NF endocytosis. This was similar to our observations in PC12 cells, where we found an apparently nonresponsive population of transfected cells that did not show increased levels of HA-NF endocytosis upon DCX overexpression. We note that our cultures also contained a population of DCX-negative cells that endocytosed NF efficiently. These might correspond to glial cells, which are known to express glial isoforms of NF, such as NF-155 (Sherman et al., 2005; Salzer et al., 2008). These observations suggested that DCX was capable of boosting neurofascin endocytosis in neurons. 
Lastly, since NF endocytosis was most robust in developing neurons ( $<$ DIV7), we wanted to know whether levels of endogenous NF at the AIS in developing neurons were more profoundly affected by downregulation of DCX than in more mature cells (DIV14; Fig. 1D). Since NF accumulation at the AIS was not extensive for about 1 week in culture (Boiko et al., 2007) and was still variable and incomplete at DIV3/4, we quantified NF levels at the AIS in DIV7 neurons after expression of shRandom or shDCX\#2 for $7 \mathrm{~d}$. We observed a significant decrease of NF at the AIS in developing neurons (Fig. $10 D$ ) that was larger than in more mature neurons. DCX levels thus modulate NF localization in developing neurons.

\section{Discussion}

DCX is genetically linked to lissencephaly, and most patient mutations map to the DC repeats in the N-terminal portion of DCX (for review, see Salzer, 2003; Liu, 2011). The best-known biochemical activity of DCX is binding to microtubules via its DC repeats. For these reasons, the emphasis in studying DCX function has been on its microtubulebinding functions. Even though other interactions in addition to microtubules have been reported, their significance for DCX function and lissencephaly are largely unknown. In this work, we explore the functional significance of the interaction of DCX with the cell adhesion receptor neurofascin. This interaction was reported several years ago (Kizhatil et al., 2002), but its role is not known.

We present evidence that (1) DCX plays a role in modulating neurofascin localization on the plasma membrane in developing neurons; (2) DCX acts by increasing endocytosis from non-AIS regions, such as soma and dendrites; and (3) the effect of DCX on neurofascin endocytosis does not require microtubule binding of DCX.

We thus propose that DCX has additional, microtubule-independent functions such as endocytosis and thereby contributes to finetuning the accumulation of neurofascin on specific areas of the plasma membrane. The roles of endocytosis and endosomal trafficking of CAMs in neurodevelopment are increasingly being elucidated (for review, see Winckler and Mellman, 2010). For instance, endocytosis of $\mathrm{N}$-cadherin and integrin has already been implicated in neuronal migration (Kawauchi et al., 2010; Shieh et al., 2011). Our work opens the exciting possibility — to be explored in the future-that DCX plays additional microtubule-independent roles such as regulating endocytosis of certain CAMs during neurodevelopment.

A new pathway for modulating neurofascin enrichment at the AIS

The AIS is of fundamental importance to neuronal function in at least three distinct ways (for review, see Lasiecka et al., 2009;
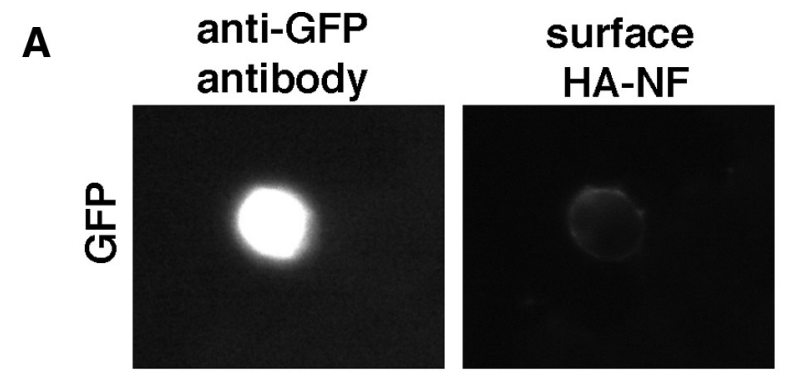

\section{endocytosed HA-NF}
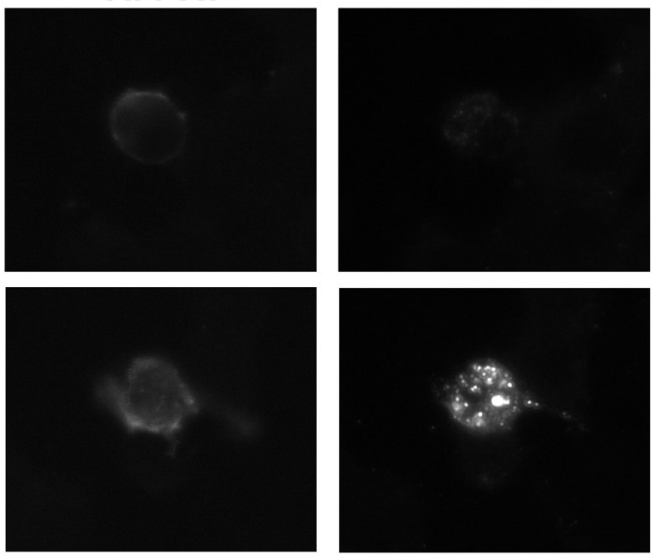

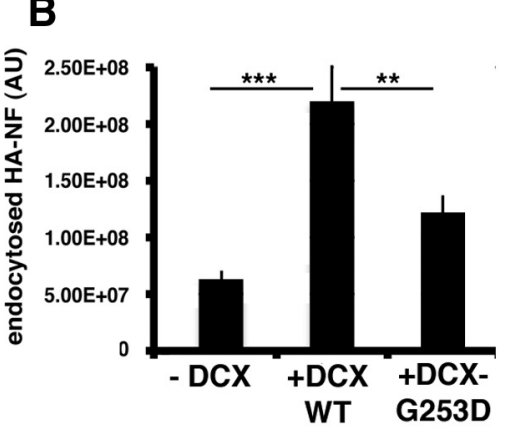

D

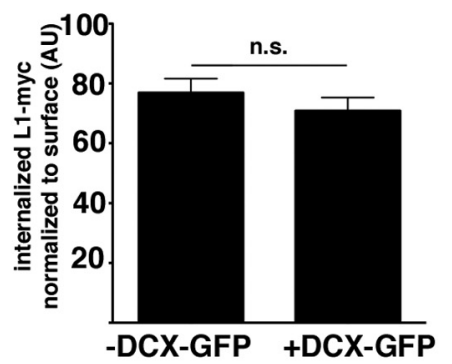

C

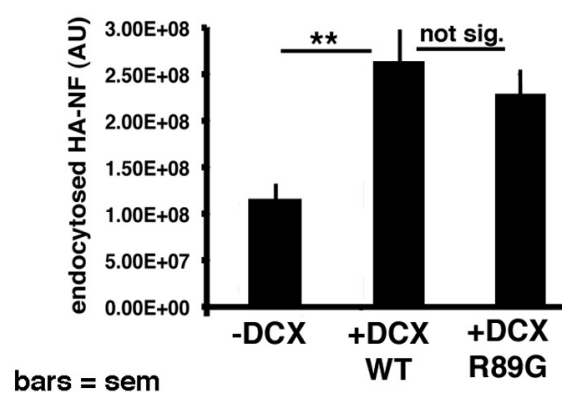

E transferrin endocytosis

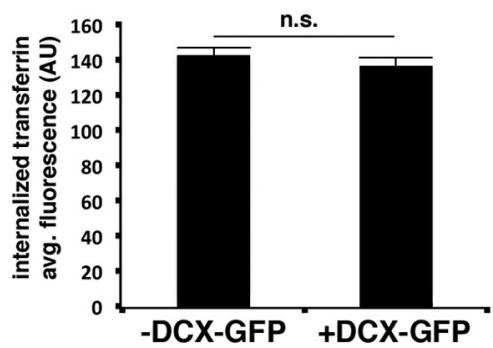

Figure 9. Endocytosis of HA-NF is stimulated by coexpression of DCX-GFP in PC12 cells. $\boldsymbol{A}, \mathrm{PC12}$ cells were transfected with HA-NF and GFP ( $\boldsymbol{A}$, top panels) or DCX-GFP ( $\boldsymbol{A}$, bottom panels) in the presence of NGF and endocytosis assays carried out with anti-HA-antibody. Individual channels for a representative cell are shown. $B, C$, Quantification of internalized HA-NF. Two to five independent experiments were analyzed. Results from one representative experiment are shown. $\boldsymbol{B}, N=59$ cells for controls, 82 cells for DCX-GFP, 84 cells for DCX-G253D-GFP.C, $N=76$ cells for controls, 91 cells for DCX-GFP, 84 cells for DCX-R89G-GFP; ${ }^{* * *} p<$ $0.0001 ;{ }^{* *} p<0.001$. D, DCX-GFP does not promote the endocytosis of L1-myc. $N=34$ cells for GFP, 32 cells for DCX-GFP. The differences are not statistically significant. $E, D C X$-GFP does not promote the endocytosis of transferrin. $N=72$ cells for GFP, 76 cells for DCX-GFP. The differences are not statistically significant. Bars, SEM.

Ogawa and Rasband, 2008): first, VGSCs clustered on the AIS enable the initiation of action potentials; second, the AIS creates diffusion barriers for membrane, cytoskeletal, and cytosolic proteins that maintain neuronal polarity; and thirdly, the AIS serves as the postsynaptic site for numerous inhibitory synapses. These features in combination thus make the AIS a crucial determinant for neuronal excitability. Our data suggest that the levels of NF at the AIS can be dynamically regulated during development downstream of neurotrophin signaling. We propose that local signaling of neurotrophins (at non-AIS regions) leads to phosphorylation of the FIGQY motif in the cytoplasmic tail of NF. The phosphorylated FIGQY motif has low affinity for ankyrin, but 


\section{A shRandom-GFP DCX endocytosed NF}
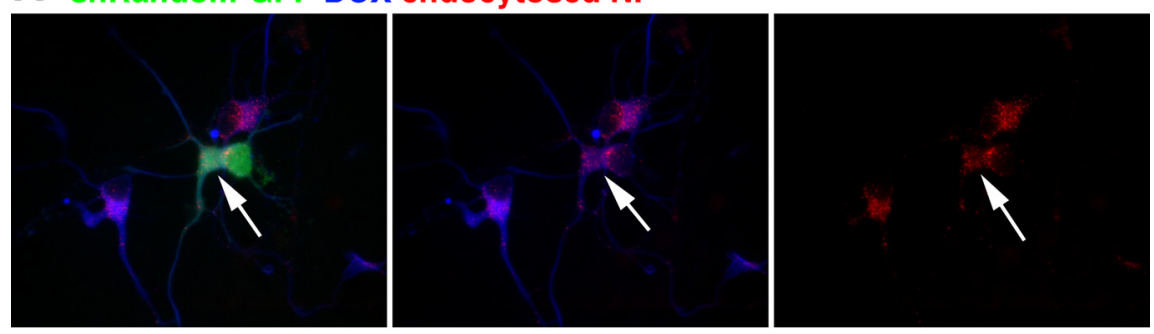

B shDCX\#2-GFP DCX endocytosed NF
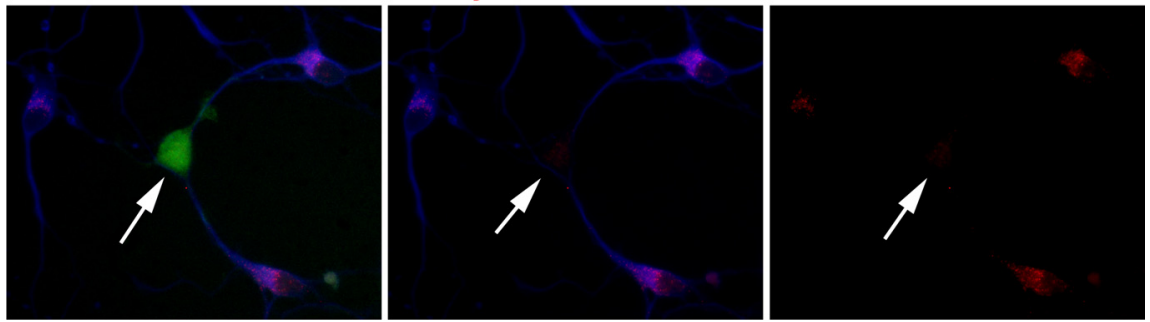

C

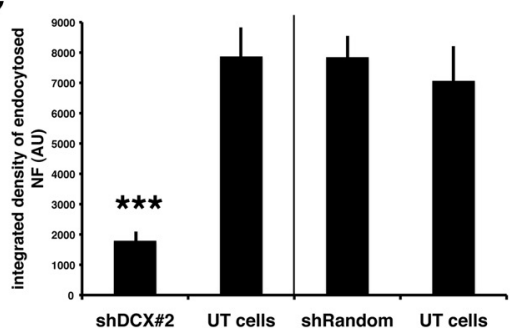

D

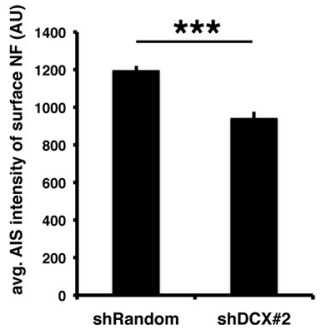

Figure 10. Endocytosis of endogenous NF is diminished after downregulation of DCX in neurons. $\boldsymbol{A}, \boldsymbol{B}$, Dissociated hippocampal neurons were electroporated with either shRandom-GFP $(\boldsymbol{A})$ or $\operatorname{shDCX\# 2-GFP}(\boldsymbol{B})$ before plating and endocytosis assays with anti-NF antibody carried out at DIV3. Cultures were fixed and stained against GFP (green), DCX (blue), and endocytosed anti-NF (red). The arrow points at the transfected cell. DCX (blue) is diminished in the cell expressing shDCX\#2-GFP ( $\boldsymbol{B}$, middle panel), but not in the cell expressing shRandom-GFP ( $\boldsymbol{A}$, middle panel). Red channel alone is shown in the right panel. The cell expressing shDCX\#2-GFP (B, right panel) shows less endocytosed NF than the untransfected cells in the same field. $\boldsymbol{C}$, The levels of endocytosed NF were quantified in cells expressing shDCX\#2 compared to untransfected cells (UT) in the same field, or expressing shRandom compared to UT cells. One representative experiment (of two independent experiments) is shown. $N=25$ cells for shDCX\#2 and 52 for corresponding UT cells; $N=24$ cells for shRandom and 26 for corresponding UT cells; ${ }^{* * *} p<0.0001$. Bars, SEM. D, AIS intensity was determined for endogenous neurofascin in developing neurons (DIV7, plasmid expression for $7 \mathrm{~d}$ ) after transfection with shRandom or shDCX\#2. $N=125$ cells for shRandom, $n=126$ cells for shDCX\#2; ${ }^{* *} p<0.0001$; Bars, SEM.

high affinity for DCX. We previously showed that phosphoFIGQY epitopes were enriched along the axon, with the highest levels at distal portions and reduced phosphoFIGQY staining on the AIS itself (Boiko et al., 2007). This observation is consistent with a model of local regulation of ankyrin- and DCX-binding capacity of NF. We propose that phosphoFIGQY-NF binds to DCX locally. NF is then endocytosed from the neuronal plasma membrane, especially in soma and dendrites. The endocytic machinery required for this event is not yet clear but may involve AP-2 clathrin adaptors. In mature cells where NF is highly accumulated at the AIS, phosphoFIGQY levels are low, presumably due to activity of a currently unidentified phosphatase. Non-phosphoFIGQY-NF can therefore bind to AIS-resident ankyrinG, thereby stabilizing NF on the AIS. In agreement with this high stability of nonphospho-NF at the AIS, we observed the highest extent of NF endocytosis in developing neurons where NF is not yet highly stabilized and accumulated at the AIS.

The postendocytic fate of NF is also not yet known, but it might involve retrograde transport and degradation or retrograde transport coupled with eventual recycling at the AIS (or elsewhere). These subsequent steps are also likely subject to regulation. It is interesting in this regard that we observed a substan- tial pool of intracellular NF in dendrites. Given that the effect of DCX depletion on AIS levels of NF is moderate ( $\sim 25 \%$ decrease), but effects on NF endocytosis are large $(\sim 4$-fold), we propose that initial delivery to the cell surface per se is not regulated by DCX, and additional pathways (including possibly direct delivery to the AIS) are responsible for some of the AIS-resident surface pool of NF. Given the high stability of NF on the cell surface (half-life on the order of $5 \mathrm{~d}$ ) (Hedstrom et al., 2007; Zhang et al., 2012), we expect a $\sim 50 \%$ reduction in NF levels in the time frame of the downregulation experiments. We estimate that the $25 \%$ effect observed in our experiment (Fig. 10D), therefore, accounts for about half of the maximal effect. The other half of NF trafficking to the AIS appears not sensitive to DCX. We speculate that it might be derived from a nonendosomal pool. The role of DCX in our model is one of a regulated modulator of NF levels and distribution in different plasma membrane domains. Given the predominant developmental expression of DCX, it likely plays a developmental role in NF endocytosis. In adult neurons, we predict that NF surface distribution is not subject to DCXmediated endocytosis and regulation.

Interestingly, Bénédicte Dargent (Université de la Méditerranée, Marseille, France) and coworkers (Brechet et al., 2008) showed that AIS targeting of voltage-gated sodium channels (VGSCs) is also regulated by phosphorylation of its ankyrin-binding site, but in the opposite manner to NF. Phosphorylation of the VGSC ankyrin-binding site increases ankyrin-binding affinity. This phosphorylation event occurs downstream of casein kinase II signaling. Neurons thus use distinct regulatory cascades to target different membrane components to the AIS. Since modulating AIS composition is a potential point for functional regulation of excitability (Grubb and Burrone, 2010; Kuba, 2010; Rasband, 2010), some of the regulatory mechanisms uncovered by us in this work for NF and by others for ion channels (Brechet et al., 2008; Vacher et al., 2011) might prove important for changing levels of crucial AIS proteins in a graded fashion to regulate excitability. More work in the future will shed light on these potential mechanisms.

\section{A role for DCX in regulated endocytosis in neurodevelopment?}

DCX is linked to lissencephaly, with surviving patients displaying mental retardation and intractable epilepsy (LoTurco and Bai, 2006; Kerjan and Gleeson, 2007). Mutations in or the absence of DCX cause major migratory defects of multiple neuronal populations during development. In addition, DCX affects dendritogenesis (Deuel et al., 2006; Cohen et al., 2008). Two genes closely related to DCX, DCLK1 and DCLK2, have partially redundant functions with DCX. Analysis of double knockout mice for DCX and DCLK1 have uncovered a previously unsuspected role for the 
DCX proteins in axon outgrowth and dendrite branching and demonstrated aberrant trafficking of synaptic vesicle proteins (Deuel et al., 2006). Mice lacking both DCX and DCLK2 show hippocampal hyperexcitability resulting in spontaneous seizures, and pyramidal neurons in the hippocampus of these mice have reduced dendritic arbors and inhibitory tone (Kerjan et al., 2009). What are the molecular mechanisms by which DCX acts in these pathways? The focus has been on DCX as a microtubuleassociated protein, in particular its ability to bind microtubules, to promote microtubule polymerization and stabilize microtubules against disassembly. Exactly what the DCX-mediated microtubule functions are is not fully understood. Interestingly, in several DCX patient mutations, in vitro microtubule-binding per se is preserved (Sapir et al., 2000), raising the possibility that DCX also acts via other mechanisms (Friocourt et al., 2003). Besides interactions with microtubules, DCX has several other known binding partners. For instance, it reportedly can bind to LIS1 (Caspi et al., 2000), itself a lissencephaly gene. LIS1 binds the retrograde motor dynein (Tanaka et al., 2004). The interaction of DCX with the dynein complex via LIS1 might contribute to the migratory defect of cortical neurons found in DCX mutants.

Several of the other reported defects in DCX deficiencies have led to suggestions that DCX is involved in vesicle trafficking. Defects in synaptic vesicles and synaptic vesicle proteins (such as VAMP2) have all been found (Friocourt et al., 2003; Deuel et al., 2006). The exact molecular mechanisms of such putative trafficking effects of DCX, though, are not known, nor are the cargos. The Bennett laboratory (Kizhatil et al., 2002) identified DCX as a binding partner of phosphoFIGQY-NF several years ago, but the biological relevance of this interaction has been undefined. Our data support the model that NF endocytoses in a DCX-facilitated fashion. In addition, microtubule binding per se is not required to support NF endocytosis by DCX. Our work thus suggests a new additional role for DCX: promoting NF endocytosis and thus regulating the relative accumulation of NF on soma, dendrites, and the AIS. In addition to the role in AIS maintenance (Zonta et al., 2011), there might be roles for NF in earlier developmental processes. In vitro, NF modulates axon outgrowth (Pruss et al., 2006), and in vivo, NF plays a role in guiding GABAergic innervation onto the soma and the AIS (Ango et al., 2004; Kriebel et al., 2011). Since we observe the most robust endocytosis of NF in young neurons, we speculate that DCX-modulation of NF levels could also play a role in developmental processes. More work is needed to test a potential role for DCX-mediated endocytosis in a developmental system.

\section{References}

Ango F, di Cristo G, Higashiyama H, Bennett V, Wu P, Huang ZJ (2004) Ankyrin-based subcellular gradient of neurofascin, an immunoglobulin family protein, directs GABAergic innervation at purkinje axon initial segment. Cell 119:257-272.

Bai J, Ramos RL, Ackman JB, Thomas AM, Lee RV, LoTurco JJ (2003) RNAi reveals doublecortin is required for radial migration in rat neocortex. Nat Neurosci 6:1277-1283.

Benmerah A, Bayrou M, Cerf-Bensussan N, Dautry-Varsat A (1999) Inhibition of clathrin-coated pit assembly by an Eps15 mutant. J Cell Sci 112:1303-1311.

Boiko T, Vakulenko M, Ewers H, Yap CC, Norden C, Winckler B (2007) Ankyrin-dependent and -independent mechanisms orchestrate axonal compartmentalization of L1 family members neurofascin and L1/ neuron-glia cell adhesion molecule. J Neurosci 27:590-603.

Bréchet A, Fache MP, Brachet A, Ferracci G, Baude A, Irondelle M, Pereira S, Leterrier C, Dargent B (2008) Protein kinase CK2 contributes to the organization of sodium channels in axonal membranes by regulating their interactions with ankyrin G. J Cell Biol 183:1101-1114.
Burkarth N, Kriebel M, Kranz EU, Volkmer H (2007) Neurofascin regulates the formation of gephyrin clusters and their subsequent translocation to the axon hillock of hippocampal neurons. Mol Cell Neurosci 36:59-70.

Caspi M, Atlas R, Kantor A, Sapir T, Reiner O (2000) Interaction between LIS1 and doublecortin, two lissencephaly gene products. Hum Mol Genet 9:2205-2213.

Cohen D, Segal M, Reiner O (2008) Doublecortin supports the development of dendritic arbors in primary hippocampal neurons. Dev Neurosci 30:187-199.

des Portes V, Francis F, Pinard JM, Desguerre I, Moutard ML, Snoeck I, Meiners LC, Capron F, Cusmai R, Ricci S, Motte J, Echenne B, Ponsot G, Dulac O, Chelly J, Beldjord C (1998) doublecortin is the major gene causing X-linked subcortical laminar heterotopia (SCLH). Hum Mol Genet 7:1063-1070.

Deuel TA, Liu JS, Corbo JC, Yoo SY, Rorke-Adams LB, Walsh CA (2006) Genetic interactions between doublecortin and doublecortin-like kinase in neuronal migration and axon outgrowth. Neuron 49:41-53.

Dzhashiashvili Y, Zhang Y, Galinska J, Lam I, Grumet M, Salzer JL (2007) Nodes of Ranvier and axon initial segments are ankyrin G-dependent domains that assemble by distinct mechanisms. J Cell Biol 177:857-870.

Fourgeaud L, Bessis AS, Rossignol F, Pin JP, Olivo-Marin JC, Hémar A (2003) The metabotropic glutamate receptor mGluR5 is endocytosed by a clathrin-independent pathway. J Biol Chem 278:12222-12230.

Friocourt G, Chafey P, Billuart P, Koulakoff A, Vinet MC, Schaar BT, McConnell SK, Francis F, Chelly J (2001) Doublecortin interacts with mu subunits of clathrin adaptor complexes in the developing nervous system. Mol Cell Neurosci 18:307-319.

Friocourt G, Koulakoff A, Chafey P, Boucher D, Fauchereau F, Chelly J, Francis F (2003) Doublecortin functions at the extremities of growing neuronal processes. Cereb Cortex 13:620-626.

Gleeson JG, Allen KM, Fox JW, Lamperti ED, Berkovic S, Scheffer I, Cooper EC, Dobyns WB, Minnerath SR, Ross ME, Walsh CA (1998) Doublecortin, a brain-specific gene mutated in human X-linked lissencephaly and double cortex syndrome, encodes a putative signaling protein. Cell 92: 63-72.

Grubb MS, Burrone J (2010) Building and maintaining the axon initial segment. Curr Opin Neurobiol 20:481-488.

Hedstrom KL, Rasband MN (2006) Intrinsic and extrinsic determinants of ion channel localization in neurons. J Neurochem 98:1345-1352.

Hedstrom KL, Xu X, Ogawa Y, Frischknecht R, Seidenbecher CI, Shrager P, Rasband MN (2007) Neurofascin assembles a specialized extracellular matrix at the axon initial segment. J Cell Biol 178:875-886.

Horton AC, Ehlers MD (2003) Neuronal polarity and trafficking. Neuron 40:277-295.

Hortsch M (2000) Structural and functional evolution of the L1 family: are four adhesion molecules better than one? Mol Cell Neurosci 15:1-10.

Kappeler C, Dhenain M, Phan Dinh Tuy F, Saillour Y, Marty S, Fallet-Bianco C, Souville I, Souil E, Pinard JM, Meyer G, Encha-Razavi F, Volk A, Beldjord C, Chelly J, Francis F (2007) Magnetic resonance imaging and histological studies of corpus callosal and hippocampal abnormalities linked to doublecortin deficiency. J Comp Neurol 500:239-254.

Kawauchi T, Sekine K, Shikanai M, Chihama K, Tomita K, Kubo K, Nakajima K, Nabeshima Y, Hoshino M (2010) Rab GTPases-dependent endocytic pathways regulate neuronal migration and maturation through $\mathrm{N}$-cadherin trafficking. Neuron 67:588-602.

Kerjan G, Gleeson JG (2007) Genetic mechanisms underlying abnormal neuronal migration in classical lissencephaly. Trends Genet 23:623-630.

Kerjan G, Koizumi H, Han EB, Dubé CM, Djakovic SN, Patrick GN, Baram TZ, Heinemann SF, Gleeson JG (2009) Mice lacking doublecortin and doublecortin-like kinase 2 display altered hippocampal neuronal maturation and spontaneous seizures. Proc Natl Acad Sci USA 106:6766-6771.

Kim MH, Cierpicki T, Derewenda U, Krowarsch D, Feng Y, Devedjiev Y, Dauter Z, Walsh CA, Otlewski J, Bushweller JH, Derewenda ZS (2003) The DCX-domain tandems of doublecortin and doublecortin-like kinase. Nat Struct Biol 10:324-333.

Kizhatil K, Wu YX, Sen A, Bennett V (2002) A new activity of doublecortin in recognition of the phospho-FIGQY tyrosine in the cytoplasmic domain of neurofascin. J Neurosci 22:7948-7958.

Kriebel M, Metzger J, Trinks S, Chugh D, Harvey RJ, Harvey K, Volkmer H (2011) The cell adhesion molecule neurofascin stabilizes axo-axonic 
GABAergic terminals at the axon initial segment. J Biol Chem 286:2438524393.

Kuba H (2010) Plasticity at the axon initial segment. Commun Integr Biol 3:597-598.

Lasiecka ZM, Yap CC, Vakulenko M, Winckler B (2009) Compartmentalizing the neuronal plasma membrane from axon initial segments to synapses. Int Rev Cell Mol Biol 272:303-389.

Leger PL, Souville I, Boddaert N, Elie C, Pinard JM, Plouin P, Moutard ML, des Portes V, Van Esch H, Joriot S, Renard JL, Chelly J, Francis F, Beldjord C, Bahi-Buisson N (2008) The location of DCX mutations predicts malformation severity in X-linked lissencephaly. Neurogenetics 9:277-285.

Liu JS (2011) Molecular genetics of neuronal migration disorders. Curr Neurol Neurosci Rep 11:171-178.

LoTurco JJ, Bai J (2006) The multipolar stage and disruptions in neuronal migration. Trends Neurosci 29:407-413.

Maness PF, Schachner M (2007) Neural recognition molecules of the immunoglobulin superfamily: signaling transducers of axon guidance and neuronal migration. Nat Neurosci 10:19-26.

Ogawa Y, Rasband MN (2008) The functional organization and assembly of the axon initial segment. Curr Opin Neurobiol 18:307-313.

Pruss T, Niere M, Kranz EU, Volkmer H (2004) Homophilic interactions of chick neurofascin in trans are important for neurite induction. Eur J Neurosci 20:3184-3188.

Pruss T, Kranz EU, Niere M, Volkmer H (2006) A regulated switch of chick neurofascin isoforms modulates ligand recognition and neurite extension. Mol Cell Neurosci 31:354-365.

Rasband MN (2010) The axon initial segment and the maintenance of neuronal polarity. Nat Rev Neurosci 11:552-562.

Salzer JL (2003) Polarized domains of myelinated axons. Neuron 40: 297-318.

Salzer JL, Brophy PJ, Peles E (2008) Molecular domains of myelinated axons in the peripheral nervous system. Glia 56:1532-1540.

Sapir T, Horesh D, Caspi M, Atlas R, Burgess HA, Wolf SG, Francis F, Chelly J, Elbaum M, Pietrokovski S, Reiner O (2000) Doublecortin mutations cluster in evolutionarily conserved functional domains. Hum Mol Genet 9:703-712.

Schaar BT, Kinoshita K, McConnell SK (2004) Doublecortin microtubule affinity is regulated by a balance of kinase and phosphatase activity at the leading edge of migrating neurons. Neuron 41:203-213.

Sherman DL, Tait S, Melrose S, Johnson R, Zonta B, Court FA, Macklin WB, Meek S, Smith AJ, Cottrell DF, Brophy PJ (2005) Neurofascins are required to establish axonal domains for saltatory conduction. Neuron 48:737-742.

Shieh JC, Schaar BT, Srinivasan K, Brodsky FM, McConnell SK (2011) Endocytosis regulates cell soma translocation and the distribution of adhesion proteins in migrating neurons. PLoS One 6:e17802.
Tanaka T, Serneo FF, Higgins C, Gambello MJ, Wynshaw-Boris A, Gleeson JG (2004) Lis1 and doublecortin function with dynein to mediate coupling of the nucleus to the centrosome in neuronal migration. J Cell Biol 165:709-721.

Tint I, Jean D, Baas PW, Black MM (2009) Doublecortin associates with microtubules preferentially in regions of the axon displaying actin-rich protrusive structures. J Neurosci 29:10995-11010.

Tsukada M, Prokscha A, Oldekamp J, Eichele G (2003) Identification of neurabin II as a novel doublecortin interacting protein. Mech Dev 120:1033-1043.

Tuvia S, Garver TD, Bennett V (1997) The phosphorylation state of the FIGQY tyrosine of neurofascin determines ankyrin-binding activity and patterns of cell segregation. Proc Natl Acad Sci U S A 94:12957-12962.

Vacher H, Yang JW, Cerda O, Autillo-Touati A, Dargent B, Trimmer JS (2011) Cdk-mediated phosphorylation of the Kvbeta2 auxiliary subunit regulates Kv1 channel axonal targeting. J Cell Biol 192:813-824.

Volkmer H, Leuschner R, Zacharias U, Rathjen FG (1996) Neurofascin induces neurites by heterophilic interactions with axonal NrCAM while NrCAM requires F11 on the axonal surface to extend neurites. J Cell Biol 135:1059-1069.

Winckler B, Mellman I (2010) Trafficking guidance receptors. Cold Spring Harb Perspect Biol 2:a001826.

Wisco D, Anderson ED, Chang MC, Norden C, Boiko T, Fölsch H, Winckler B (2003) Uncovering multiple axonal targeting pathways in hippocampal neurons. J Cell Biol 162:1317-1328.

Yap CC, Wisco D, Kujala P, Lasiecka ZM, Cannon JT, Chang MC, Hirling H, Klumperman J, Winckler B (2008a) The somatodendritic endosomal regulator NEEP21 facilitates axonal targeting of L1/NgCAM. J Cell Biol $180: 827-842$.

Yap CC, Nokes RL, Wisco D, Anderson E, Fölsch H, Winckler B (2008b) Pathway selection to the axon depends on multiple targeting signals in NgCAM. J Cell Sci 121:1514-1525.

Yap CC, Lasiecka ZM, Caplan S, Winckler B (2010) Alterations of EHD1/ EHD4 protein levels interfere with L1/NgCAM endocytosis in neurons and disrupt axonal targeting. J Neurosci 30:6646-6657.

Zhang X, Davis JQ, Carpenter S, Bennett V (1998) Structural requirements for association of neurofascin with ankyrin. J Biol Chem 273:3078530794.

Zhang Y, Bekku Y, Dzhashiashvili Y, Armenti S, Meng X, Sasaki Y, Milbrandt J, Salzer JL (2012) Assembly and maintenance of nodes of ranvier rely on distinct sources of proteins and targeting mechanisms. Neuron 73: 92-107.

Zonta B, Desmazieres A, Rinaldi A, Tait S, Sherman DL, Nolan MF, Brophy PJ (2011) A critical role for Neurofascin in regulating action potential initiation through maintenance of the axon initial segment. Neuron 69:945956. 